\title{
MATHEMATICAL MODELS, PROBLEMS, AND CONTROVERSIES OF EVOLUTIONARY THEORY ${ }^{1}$
}

\author{
BY SAMUEL KARLIN
}

1. The year 1982, the centenary of Charles Darwin's death, witnessed conferences, symposia, and publications in many disciplines devoted to reassessing the theory of evolution by natural selection and all its ramifications. A brief historical review on developments in evolutionary theory emphasizing the role of mathematics is given in $\$ 3$ of this paper. $\$ 2$ presents background material on agents of evolutionary processes. $\$ 4$ contains an annotated listing of several problems and controversies pertaining to evolutionary processes, and a more extended discussion of the neutralist-selectionist controversy. $\$ 5$ presents background information relating to sex ratio in various plant and animal species. Several classes of mathematical models of much recent interest centering on sex ratio evolution and related dynamical systems are examined in $\S \S 6-8$; the purely mathematically oriented reader can pass directly to these sections. The concluding section presents our views on several philosophic issues in evolutionary theory today, and on the nature of the mathematics relevant to the genetics of the current decade.

Taxonomists have recorded 1-2 million plant and animal species and surmise that another 4-6 million species remain to be classified. More than $50 \%$ of all living species are of the insect genera; the Drosophila flies alone comprise at least 2,500 species. Counts of bird species vary from 8,000 to 10,000 . The number of species from the evolution of life to the present is estimated at 4-16 billion. Simpson (1953) projects that more than $99.9 \%$ of all species that ever existed are extinct.

The ubiquitous variability within species of biochemical, morphological, physiological, and behavioral traits is also intriguing. As observation, and experimental techniques are being refined, increasing numbers of segregating genes (those exhibiting at least two alternative types) are being detected. Another aspect of nonuniformity is the prodigious variety of sexual mechanisms, mating patterns, life cycles, life styles, strategies for survival and reproduction, growth characteristics, and ecosystem interactions.

Concomitant to the observed diversity in living forms and life patterns, there are some universals and expressions of identity. The basic DNA-RNA structure and its replication mechanism are present in "all" organisms. Proteins

\footnotetext{
The Josiah Willard Gibbs lecture was presented at the 89th annual meeting of the American Mathematical Society in Denver, Colorado, January 5, 1983; received by the editors October 5, 1983.

1980 Mathematics Subject Classification. Primary 92A10, 92-02; Secondary 92-03.

${ }^{1}$ Supported in part by NIH Grant 5R01 GM10452-20 and NSF Grant MCS79-24310.
} 
appear to be an essential component of life. And though energy conversion and production are managed in a number of ways, common to all these ways is the generation and use of ATP (Adenosine Triphosphate), the main energy source used in driving all other activities.

Many evolutionists contend that both the immense diversity of life and its universals are products of the Darwinian principle of natural selection, which operates on genetic variation in responding to the challenges of ever-changing environmental conditions.

A central problem of evolutionary theory is to explain the vast variability observed on all levels from the molecular to organizational characteristics. How much of this variability and what kinds can be accounted for by natural selection, and what is the nature of the selection forces and causal mechanisms?

2. Agents and forces of evolutionary processes. We begin by defining some terms. Chromosomes, usually found in the nucleus, mostly govern the inheritable characteristics of an organism. Chromosomes may occur singly (the haploid case), as in bacteria and some fungi; in pairs (the diploid case), as in mammals; or in larger groups (triploid, tetraploid, in general polyploid), as in many plants. The associated pairs, triplets, etc. are called homologous chromosomes. Locus is the position at which a gene (a sort of unit of the chromosome) occurs on a chromosome. Alleles are alternate gene forms at a given locus. Genotypes are the various possible combinations of alleles at corresponding loci on homologous chromosomes. In the diploid case if the alleles are $\mathrm{A}$ and $\mathrm{a}$, the genotypes are AA, Aa, and aa.

The populations to be considered, unless specified otherwise, contain diploid individuals. We shall assume that an offspring is formed by the donation of a gamete (one of each pair of homologous chromosomes) from each of two parents. In the case of one locus, each parent, depending on its genotype, may donate either $\mathrm{A}$ or a to form a zygote (fertilized egg) of genotype AA, Aa, or aa. Individuals with genotype $\mathrm{AA}$ or aa are homozygotes; those with $\mathrm{Aa}$ are heterozygotes. The physical manifestation of the genotype is called the phenotype.

Consider the case of two loci, where the alleles $\mathrm{A}$ and a are possible at the first locus and alleles $B$ and $b$ at the second locus. A typical one of the ten possible genotypes could be written $\mathrm{AB} / \mathrm{ab}$, which signifies that $\mathrm{AB}$ are linked on one chromosome with $A$ at the first locus and $B$ at the second and that $a b$ are correspondingly situated on the second chromosome. Recombination may occur in the case of two loci, and the two loci are not independent so far as gamete donation is concerned. An individual heterozygous at both loci can produce four types of gametes. For example, an individual of genotype $A B / a b$ can produce gametes of types $\mathrm{AB}$ and $\mathrm{ab}$ and also gametes of types $\mathrm{Ab}$ and $\mathrm{aB}$. The $A B$ and $a b$ gametes are called parental, while $A b$ and $a B$ are called recombinant. If the loci are linked, there will be an excess of parental gametes over recombinants. It is generally found that the parental types $A B, a b$ are produced with equal frequencies $\frac{1}{2}(1-r)$ and the recombinant types with equal frequencies $\frac{1}{2} r$, where the number $r, 0<r \leqslant 1$, is called the recombination fraction. 
For further information and biological scope on these terms, consult, for example, the texts by Futuyma (1979), Hartl (1980), Wallace (1981), and Hedrich (1983).

Causal and historical analyses of the evolutionary process fall back on a combination of modeling, interpretation and systematization of the paleontological record, experimental findings, field observation, and data on the phylogeny of the living world. The agencies acting on populations of individuals include natural and sexual selection forces, mutation and migration pressures, mating patterns, interaction of genes, recombination and linkage relations, changing environmental conditions, the influence of the initial population makeup, historical factors, and a conglomeration of chance factors. We now briefly discuss these factors in qualitative terms.

Natural selection. Fertility, viability, and segregation distortion are the major representations of what Darwin called "fitness". Differential viability refers to how the types differ in their ability to survive and reproduce. Fertility selection reflects the variation in numbers of offspring produced by the different parental crosses. Segregation distortion (known also as meiotic drive) refers to deviation from the Mendelian segregation ratio, in which both parents contribute equally to the offspring.

Mutation. Mutation events, the ultimate source of genetic variability, occur constantly. In many species mutation at the gene level comes about by substitution, deletion, or addition of mucleotides in DNA chains. These can alter enzyme function or change the time, place, or amount of protein synthesis. Chromosomal mutations and aberrations change the arrangement of genes or duplicate blocks of genes, or translocate and bind segments of chromosomal pieces, etc. The location of mutation events may be finely or coarsely tuned. The rates of mutation vary among species and are sensitive to environmental conditions. Some modes of mutation rate may be under genetic control. Mutant types tend to be deleterious, although some mutation events can produce advantageous forms.

Migration and population structure. The mixing of populations is an important consideration in explicating spatial frequency patterns of various traits. The concept of subpopulation primarily refers to separate breeding units differentiated by a myriad of social, geographical, and historical criteria. Population structure (how individuals are distributed and mate) depends to a large extent on the pattern of migration, age classes, and behavioral and physiological endowments (which may not be constant in time or space).

Mating patterns and sexual systems. The variety of mating systems in nature is staggering. Parthenogenesis (asexual reproduction) is found pervasively in bacterial populations, among some classes of apomictic plant species (e.g., some dandelions), and in certain insect, fish, and reptile populations. Many fish species are sequential hermaphrodites, acting as males during part of their life and females during the other part. Most plant populations are simultaneous hermaphrodites, carrying both sex organs and often indulging in selffertilization. However, in a number of plant varieties, incompatibility mechanisms (analogous to separate sexes) compel total outcrossing. Most species of snails are hermaphrodites with self-fertilization prevented, but then two snails in reproduction act as both male and female with respect to each other. Among 
the social insects (e.g., wasps, bees, ants) three classes of individuals are present: fertile males (mostly haploid), fertile females, and sterile females. For mammals separate sexes is the rule.

Mating behavior can exhibit a variety of forms. By "sexual selection" Darwin meant the consequences of mating behavior. The phenomena of sexual selection complement and interact with natural selection forces in the evolutionary process.

Recombination and sex. A significant source of new variation is the phenomenon of recombination, in which separate (usually homologous) chromosomes exchange portions of DNA at reproduction (meiosis). Sexuality is a case of chromosomal recombination at the rate of $50 \%$.

Many organisms encompass in their life cycle both a sexual stage involving recombination events and an asexual vegetative stage. The recombination frequency tends to be less in males than female. Recombination is often under genetic control, occurring at specific recombination sites.

Tied to the recombination process is the extent of ploidy. Fungi live most of their lives as haploids and take excursions as diploids. Mammals are invariably diploids so that a gene unit carries two doses of the genetic material determining its genotype. Higher ploidy abounds in plant populations; it is seemingly rare in the animal kingdom. Prokaryotes (organisms whose cells lack a distinct nucleus) allow recombination from time to time mediated by proccesses of transformation (e.g., exchange of DNA between distinct bacterial strains), transduction (e.g., employing appropriate vector viruses to transfer DNA material between species), and conjugation (a process akin to sexuality).

Environmental factors. The interactions of natural and sexual selection forces, mutation and migration pressures, and environment are complex and subtle. Many things need to be considered, e.g., the effects of frequency- and density-dependent factors, the age structure of the population, behavioral characteristics, life history strategies, ecological variables, species abundancies, and historical factors. The aspect of changing environments confounds individual and population "adaptations". The undeniable fact that environments persistently involve both systematic and randomly changing elements adds a large unpredictable aspect to the study of the life sciences. But as Paul A. Samuelson (1976, p. 120) states, "The art of science is to infer the invariance aspects of that which is ever changing."

Small population size, chance factors, historicity, and initial conditions. A number of geneticists place much emphasis on the importance of finite (small) population size in bringing changes during the evolutionary process. In a small population this force (known as "sampling effects" or "random genetic drift") refers mainly to statistical (uncontrolled) variability, that is, to chance fluctuations associated with merely reproducing the population numbers over successive generations. In this situation a deleterious mutant type can be established purely by chance.

There are many polemics in the evolutionary literature between those who emphasize the role of history, the uniqueness of events, and the existence of equilibrium states and those who claim that practically all population configurations reflect transient states and emphasize the importance of chance as opposed to the determinism of natural selection. 
Some claim, on the basis of paleontological and other geological data, that important evolutionary changes occur at population "bottlenecks", times of sudden reduced population sizes.

Chance and randomness enter into the picture independently of sampling perturbations by virtue of the constantly changing environment. A "realistic" model entails the parameters of selection, mutation, migration, and mating patterns as coupled random processes. The frequency outcomes are accordingly also random processes, even where population size is large and "random genetic drift" is inconsequential.

Multigene interactions. In a population we can consider traits determined at a single locus or several loci, or traits of a polygenic kind. Most red blood serum typings (e.g., the $A B O$ system, $R h$ ) and many disease susceptibilities (e.g., Albino trait, Tay Sachs disease) are determined at a single locus.

Some sets of loci apparently arise from the duplication of a single gene and subsequently develop partially differentiated functions while retaining strong homology. Thus myoglobin (a protein that facilitates the storage of oxygen in muscles) and the multigene family of hemoglobin proteins (molecules in red blood cells that transport oxygen from the lungs) are both presumed to derive by duplication from the same remote ancestor gene complex.

A polygenic or quantitative character refers to a trait determined by many loci (genes) contributing mostly small effects and commonly manifesting a continuous variation in the trait expression.

The analysis of multilocus systems is essential to understanding the nature of epistasis (the interaction of selective effects between genes) and linkage (the extent to which genes influencing a given character are located together). Equilibrium, adaptation, speciation, development, and differentiation all necessarily involve the interaction of genes.

Sources of data. A primary source of evolutionary data is furnished by the fossil record; another is field observations by naturalists. In the past, polymorphic genes were identified mostly in visible traits, notably color, patterns, bands in chromosomes, and a vast array of mutant markers. Recent advances in molecular biology provide new means of discerning alternative forms in populations on the biochemical level. In particular, the use of electrophoresis, chromotography, cell fusion, immunological procedures, and more elaborate separation techniques for determining molecular weight, charge, and fluorescence have produced a wealth of data pertaining to molecular population variability.

\section{A brief look at the history of theoretical and mathematical evolutionary science.}

Before 1900. The scope and meaning of "evolution" and the nature of natural selection set forth by Darwin are often interpreted in different ways among humanists, theologians, philosophers, and scientists. Darwin's writings themselves offer an enthralling experience in observation, synthesis, inference and speculation. Darwinism (the theory of natural and sexual selection) is not a scientific law capable of rigorous proof. But there is no doubt of the existence of "natural selection" underlying many changes in the composition of natural populations at all levels. 
The significance of Mendelism (although Mendel's celebrated publication appeared in 1865) comes to the fore only in the 20th century. Biological science prior to the mid-19th century primarily focused on introducing some sort of order into the classification of plants and animals. There was little quantitative theory and little qualitative speculation about the cause of the vast observed variability of forms and species.

In the latter part of the 19th century, genetic studies concentrated on plant breeding (horticultural practices). Investigators were bemused by the subtleties and variations manifested in quantitative characters, but were curious about the frequency changes of continuous traits as transmitted over successive generations in human populations. Francis Galton (a cousin of Darwin) and Karl Pearson (a protégé of Galton, trained originally as an economist) developed the fundamentals of biometry, the precursor of modern statistical science, motivated mainly by problems of evolution and eugenics. In 1889 Galton proposed that human stature is inherited and calculated the parent-offspring correlation as .33 . He also was the first to propose the use of twin studies for the purpose of assessing genetic and environmental correlations of polygenic traits.

As a model of multifactorial inheritance Galton proposed

$$
X_{n+1}=h \frac{X_{n}^{\prime}+X_{n}^{\prime \prime}}{2}+\xi_{n}+a \mu_{n},
$$

where $X_{n}^{\prime}$ and $X_{n}^{\prime \prime}$ are the parental trait values in generation $n, X_{n+1}$ is the trait value of an offspring, $\xi_{n}$ is an independent residual environmental contribution of mean $0, \mu_{n}$ is the population mean of generation $n, h^{2}$ is the heritability coefficient (the regression of an offspring on the midparental value at an equilibrium state), and $a$ serves as a constant scale adjusting the relative influence of the population to that of parental transmission. The analysis of (3.1) led to the principle of regression to the mean, according to which children resemble their parents but regress toward the population average-a concept apparently in contradiction to Mendelian principles. Pearson rejected Mendelism in a landmark publication (1904). But his work served decisively in connecting biometrical population genetics and Mendelian principles.

Galton and Pearson formalized and quantified concepts such as "population", "measures of variability", and "regression structures". They emphasized the fundamental existence of a variety of kinds of variability within and between populations, a concept difficult to comprehend in those days. The recognition of variability within populations led to the natural inquiry about the mechanisms causing this variability.

1900-1920. The year 1900 witnessed the formal recognition and rediscovery of Mendel's work by three independent plant physiologists. Mendel's famous paper of 1865 , often cited as a gem of statistical and theoretical modeling, presents a compelling mixture of experiment, observation, data analysis, deduction, and abstraction. Mendel's approach to the study of inheritance was to work with well-defined, clear-cut, discrete differences between members of a species rather than such continuous quantitative attributes as height and weight variations. 
The next 10 years (1900-1910) witnessed the application of the deductive-inductive mathematical statistical method to plant breeding and cytological data. The researchers of this time developed a renovated form of Mendelian principles and simultaneously elaborated a whole panoply of evolutionary genetic concepts. The concepts "expression of a gene", "genotype", "phenotype", "dominance", "equilibrium", "recombination", "linkage groups", "gene mapping”, “mutation”, “epistasis", “heterosis”, “inbreeding”, and "hybrid vigor” as causes and agencies of evolution were crystallized and clarified. For example, the linear order of genes was inferred by a mixture of cytological and statistical analyses.

1915-1955. Between 1915 and 1950 theoretical evolutionary genetics was dominated by the names of Sewell Wright (the Gibbs Lecturer of 1941), R. A. Fisher, and J. B. S. Haldane.

Fisher trained as a mathematician (he was a Wrangler at Cambridge) and served in his early career partly as a statistical consultant for biologists. His first efforts in genetics produced a classic paper (1918) that embodied the seminal ideas of ANOVA (analysis of variance) and aspects of the design of experiments.

The condition for selection balance at a single gene locus involving two alternative alleles, called the overdominance principle, was modeled by Fisher in 1922. This important result provides under random mating the simplest mechanism for the existence of a stable polymorphism arising solely from the balance of differential viability effects. More precisely, for random mating where the heterozygote (Aa genotype) has superior viability fitness to both homozygotes (the AA and aa genotypes) a stable polymorphism will result. This analytical finding has effectively been used to explain the gene frequency patterns of the sickle cell trait, the polymorphism of Thalassemia, and the gene frequency distribution of G6PD deficiency manifested in certain Mediterranean populations.

Modern statistical theory owes much to Fisher's efforts to design and analyze experiments to settle problems in genetics. $\mathrm{He}$ is the indisputable founder of the theory of experimentation. His efforts to understand evolutionary and genetic theory paralleled and nurtured his statistical exploits. Fisher's monograph on natural selection (1930) is still the point of departure for many developments in theoretical population genetics today.

Haldane, a colorful personality whose undergraduate degree was in classics at Oxford, did important work in the sciences, history, and politics, and wrote popular science as well. He was competent in mathematics as well as in chemistry and genetics, and contributed significantly to enzyme kinetics, statistical practice, and population biology. In a famous series of papers entitled Mathematical contributions to the theory of natural selection that appeared in the Proceedings of the Cambridge Philosophical Society in the 1920 's, Haldane set forth a variety of simple mathematical analyses concerned with the way natural selection might be supposed to act. He worked out the theoretical effects of different forms and intensities of selection and mutation on frequencies of autosomal (a gene not located on the $\mathrm{X}$ chromosome), 
dominant, recessive, and sex-linked genes. His model of mutation selection balance is important today in the study of genetic diseases and medical genetic counseling. It gives an estimate of equilibrium values where recurrent mutations of deleterious alleles are balanced by their elimination through selection. Through these models the first estimates of the mutation rate of a deleterious gene in a human population were given (Haldane (1927)).

Wright (1923a), a zoologist by training, used equilibrium principles in comparing observation with expectation, rejecting a one-gene hypothesis for the inheritance of blue eye color in man and in a case of color inheritance in cattle. (Felix Bernstein, a noted mathematician, employed in 1925 similar ideas to reach a correct interpretation of the inheritance of the $\mathrm{ABO}$ blood typings.)

Although measures of inbreeding depression were introduced earlier, it was Wright and Fisher who substantially extended the theory of systems of matings between relatives, such as those used by animal and plant breeders. Wright(1923b) devised a method of "path coefficients" to deduce the consequences of Mendelian heredity under different systems of matings. He also introduced a "coefficient of inbreeding" extending the equilibrium formulation to include a mixture of random and regular inbreeding mating patterns.

In a seminal paper (1931), Wright established that in small populations evolutionary theory should take account of the sampling effects involved in producing one generation from the previous. He called this effect "random drift". The significance of Wright's sampling force has recently become a focal point of a sharp controversy on the nature of the evolutionary process. Evolution can be considered to be a sequence of gene replacement processes, whereby in each such process one allele is replaced in a population by another allele. Classical Darwinian selectionist theory maintains that the replacing allele is superior to the replaced allele and that the mechanism directing the replacement procedure is natural selection. To the contrary, the essence of the neutralist theory is that a large proportion of the replacement process (especially on the biochemical level) takes place by virtue of chance phenomena acting on selectively equivalent alleles; that is, the new allele is no better than the old, but has arisen by mutation and increased in frequency because the individuals carrying this allele happened to leave more offspring than the remaining individuals. Thus, changes due to random genetic drift in a small population could be nonadaptive. The shifting balance theory of evolutionary chance (Wright (1968)) asserts that the makeup of local populations can move from one stable equilibrium configuration to another ("from one peak to another peak through valleys") just by sampling effects.

While the theoretical constructs of population genetics were being elaborated in this period, experimentalists and naturalists were observing and studying many genetic traits in natural populations and under laboratory conditions. A review and synthesis of observations on Mendelian populations appeared in Dobzhansky (1951). Also relevant are the works of Mayr (1963), Simpson (1953), and Stebbins (1950), among others.

1955-1980. The main directions of recent research in evolutionary theory are multilocus studies, studies of the variation of natural populations in space 
and time, the study of stochastic genetic models, the study of mixed genetic and ecological systems, and studies on the population genetics of behavioral traits.

Multilocus studies. The last 30 years have witnessed extensive theoretical studies of complex genetic systems integrating the interactive effects of several agents and relations among loci. For a review of two-locus cases, consult Hedrick et al. (1978); for studies of n-locus selection models, see Karlin (1979a) and Karlin and Avni (1981).

The last decade has also witnessed a renewal of activity devoted to the elaboration of various dynamic models of polygenic and quantitative characters. A strong motivation stems from interest in the heritability properties of behavioral, physiological, and medical measurements, e.g., coronary risk factors and cognitive traits such as IQ scores. The fact that selection, primarily for polygenic characters, acts on phenotypes, whereas segregation involves genotypes, points up the necessity for dealing with an array including both genotypes and phenotypes. Recent theoretical and mathematical models of multifactorial transmission occur in many writings, among them Lande (1975), Karlin (1979b), Bulmer (1980), and Turelli (1984).

Variation in natural populations in space and time. Environmental and/or geographical variation in selection patterns and its coupling with gene flow are considered vital ingredients in speciation and differentiation. Recent literature has witnessed the formulation and analysis of models designed to understand in more precise terms the interaction between spatial and temporal selection variation and population structure. For reviews, references, and extensive modeling results, see Felsenstein (1976), Hedrick et al. (1976), Endler (1977), Nagylaki (1978), and Karlin (1982b).

The study of stochastic genetic models. Many advances in the theoretical description of random genetic drift have been secured with the help of results and techniques of diffusion stochastic processes.

First, Wright (1931) and Malécot (1948), and later Kimura (1957, 1964, 1983) and many others extensively applied diffusion analysis to the study of stochastic genetic models; for examples see Ewens (1979) and Karlin and Taylor (1981, Chapter 15). The problems considered include the analysis of random sampling effects due to small population size, the balance in small populations of recurrent mutation and random genetic drift, calculations of the number of mutants maintained in a population, the time to detection of a mutant deleterious gene, conditioned diffusion processes and fluctuating selection intensities over successive generations due to random and/or systematic environmental changes. Feller (1951) was intrigued by the fact that the diffusions arising in genetics exhibit singularities at the boundaries.

The neutralist selection controversy (see \$4) has stimulated the study of many natural stochastic models including the infinite alleles model (Kimura and Crow (1964)), the Ewens sampling formula (1972) and its intimate connections with Poisson-Dirichlet processes (for an elegant treatment containing several perspectives, see Kingman (1980)), the concept of the age of a stochastic process and its ramifications (e.g., Kimura and Ohta (1973), Pakes and Tavaré (1981)), charge state models (Ohta and Kimura (1973)), infinite 
sites models (Watterson (1975)), wandering profile models (Moran (1975, 1976), Kingman (1976)), and genealogical stochastic structures (Kingman (1982a, b), Tavaré (1984), Watterson (1984)).

Mixed genetic and ecological systems. Three individuals pioneered the subject of ecological theory in the early 1920's: A. J. Lotka, V. Volterra, and V. A. Kostitzin. In 1925 Lotka described the interaction between two species by a differential equations system involving quadratic terms, showing that predation and parasitism could be explained by periodic changes in the species numbers. He also considered more complex "food web models".

Volterra was an accomplished mathematician who contributed significantly to the theory of integral equations before he engaged the study of some biological systems. He was attracted to mathematical ecology by the proddings of his son-in-law, a zoologist, who was anxious to explain curious variations in fish catches in the Adriatic. Volterra's competition models, similar to Lotka's, provided causal correlates for his son-in-law's observations.

Kostitzin, of Russian birth, worked mostly in France. His interest in mathematics and ecology was kindled in 1930 when he heard Volterra's lectures on The mathematical theory of the struggle for life, published in 1931. Kostitzin's textbook (1937) extends Volterra's treatment of competition and predation by including models of symbiosis and parasitism, taking account also of some genetic factors. The work of Lotka, Volterra, and Kostitzin went unrecognized for a decade and was not revived as a subject for study until the 1950's. In the late 1950's McArthur introduced the concept of resource utilization and production functions, which provided a class of competition coefficients accessible to measurement and interpretation.

The interaction of ecological and genetic systems has become a major focus of recent evolutionary studies, e.g., Roughgarden (1979), Matessi and Jayakar (1976a), and Ginsberg (1983).

Studies in the population genetics of behavioral traits. Attempts have been made to quantify the evolution of behavioral traits in the areas of group selection, kin selection, and the evolution of altruism. Theoretical and qualitative modeling along these lines have been done by Wynne-Edwards (1962) and Hamilton (1964), and mathematical formulations have been proposed by Eshel (1972), Boorman and Levitt (1973, 1980), Matessi and Jayakar (1976b), Michod (1982), and Karlin and Matessi (1983). Observation, speculation, and theorizing about behavioral patterns, organizational structure, and the like by ethologists and naturalists such as Tinbergen, Lorenz, E. O. Wilson, and G. C. Williams have been of great help in understanding the structure of certain animal and insect societies. In this literature the genetic basis of population control, mimicry, signaling and alarm calls in prey-predator situations, communication systems, and hierarchical status in groups are discussed in terms of strategy analysis, and the role of kin selection is underscored. The recent synthesis on sociobiology by E. O. Wilson (1975) has stimulated a torrid controversy on the reliability and applicability of this new framework.

In completing this brief account of the principal developments to date in theoretical population genetics, we may speculate about the implications for evolutionary theory of the remarkable recent successes in molecular biology. In 
1944 the hereditary material DNA was identified; next came the double helix model of the DNA replicating machinery, and 15 years later the universal code delineating the correspondence of nucleotide triplets to amino acid protein residues was firmly established. Thereafter the nature of mutation events in terms of DNA changes could be ascertained in concrete terms. Some of the enzymes involved in recombination have been isolated and purified, and a variety of structures of proteins and enzymes and forms of gene expression, particularly in bacterial organisms, have been dissected.

Molecular biology and biochemistry deal mostly with maintenance properties of cells as exemplified by development, differentiation, regulation, and metabolic processes. The variety and abundance of the mechanisms, and their redundancies and complexities, are of a much higher order of magnitude than had been expected. There appear to be a host of mechanisms that can adapt to the same task (perhaps not always having the same efficiency), exhibiting malleability and flexibility in response to all kinds of genomic or external environmental changes. With all the advances in molecular biology, the major problems of population genetics and evolution remain intact. How do genes contribute to fitness and interrelate in function and in response to ecological conditions? How do we relate genotype and phenotype? With respect to genetic architecture and quantitative inheritance, what are the numbers of genes involved, where are they, and what are their relative effects? These and many other problems still remain. Molecular biology has pointed to new areas of inquiry and provided vast new resources for acquiring data with which to study the degrees and forms of genetic variability, to construct phylogenic relationships between populations, and to assess the nature of selection effects.

Geneticists at the close of the last century were concerned mostly with continuously distributed traits. With the rediscovery of Mendel they took up the study of discrete traits induced by one or a few genes, and beginning in the 1950's they increasingly turned to the study of multigene interactions. In effect, then, population genetic theory has come full circle, engaging with new vigor the study of the transmissibility characteristics innate to continuous phenotypic variation over space and time.

4. An overview of problems and controversies in quantitative evolutionary theory. Two opposite tendencies operate on natural populations: natural selection, or the propensity to adapt to a given environment; and polymorphism, or the propensity to produce variation to cope with changing environments. Muller (1929) sees natural selection as a sieve that selects and retains the most fit type in a given environment. Dobzhansky (1951), by contrast, emphasizes the multifarious ways in which natural selection interacts with the challenges of the environment. Mutation and recombination events provide the system with the randomness that is needed to preserve a population in new environments where only recombinant or mutant types can survive.

All we know about evolution suggests that the evolutionary process has opted prudently for a balance between determinism and polymorphism... or, in more old-fashioned terms, conservatism and change, heredity and mutation, stability and novelty, security and incentive. 
Let us now consider whether the forces and agents discussed in the previous sections contribute to fostering increased variability or diminished variability in the content of the gene pool.

Agents promoting increased variability. (a) selection favoring heterozygotes; (b) mutation selection in opposition; (c) migration selection balance and population subdivision; (d) general multilocus selection balance (polymorphism resulting from interactions among genes and from recombination); (e) frequency-dependent selection (for example, favoring the rare type); (f) some forms of sexual selection (preferential matings); (g) incompatibility or self-sterility mechanisms; (h) varying environmental conditions including fluctuating selection intensities; (i) group selection in subdivided populations; (j) cultural transmission.

Agents promoting diminished variability. (a) gametic haploid selection; (b) directed natural selection favoring a single type; (c) disruptive selection favoring extreme types in a population; (d) self-fertilization and parthenogenesis; (e) positive assortative mating; (f) regular inbreeding systems including sibmating, parent-offspring mating, imprinting, and consanguineous mating; (g) small population size; (h) constant and/or stable environments.

Table 1 presents an annotated list of current issues and controversies of evolutionary theory to which mathematical models have been applied.

New molecular techniques have disclosed a great deal of hitherto unsuspected genetic variation on the biochemical level. Through electrophoresis, it is possible to detect single amino acid substitutions in proteins discriminated on the basis of electric charge or size. Still other genetic variations have been revealed by immunogenetic techniques for identifying antigen antibody associations.

Recent advances in DNA sequencing methodology, which have led to the identification of more than 80 restriction enzymes that cleave DNA at specific recognition sites, have uncovered an abundance of restriction-fragment-length polymorphisms (i.e., polymorphisms with respect to DNA segments cut out by these restriction endonucleases). This adds a new dimension of variability, such that one such polymorphism is predicted to occur on average every 500 nucleotides.

There is another component of variability whose measurement should be feasible in the near future: contrasts in the actual DNA composition of proteins (places and numbers of changes) between species and between individuals in a population.

The selectionist-neutralist controversy. With the explosion of data reporting polymorphism on the biochemical level, the long-standing problem of the relative importance of nonrandom and random processes in the genetic structure of populations has been revived in the form of the selectionist-neutralist controversy.

The neutralists assert that most of the molecular variation in natural populations is selectively neutral, i.e., that the various gene products of alternative variant polypeptide forms (allozymes) are equally suited to the requirements of normal development, survival, and reproduction. Accordingly, there is a continuous flux of mutation occurrences kept in small populations at low frequencies by virtue of sampling fluctuations. 
The neutralists do not deny the existence of cases of balancing selection forces, but they contend that most molecular variation has little to do with adaptive evolution. They recognize the evolution of one-locus polymorphic traits depending on disease susceptibilities (e.g., malaria promulgated by insect vectors), by factors of crypticness or mimicry to avoid predators, and by other environmental considerations. These traits include the sickle cell trait, Thallasemia, color forms in butterflies, banding in snails, and melanism.

TABLE 1. Controversies and Problems of Evolutionary Theory

1. Selectionist hypothesis vs. neutralist hypothesis of molecular evolution: more generally, the role of chance vis-à-vis determinism in evolutionary processes.

2. The nature of evolutionary change: sudden vs. gradual; punctualism vs. stasis in speciation events, trends, species selection, adaptive vs. accidental speciation.

3. Quantitative inheritance: major-gene effects compared to many-small-genes (polygenic) effects.

4. Genetics vs. environment: nature-nurture controversy, biological versus cultural inheritance and interactions.

5. The evolution of behavioral traits and altruism: group-kin selection vs. individual selection.

6. Heterogeneous environment "entails more or less variability". What is the nature of genotypic frequency distribution in a varying environment (e.g., spatial vs. temporal)?

7. Do sex and recombination accelerate or slow evolution? How can an even sex ratio be maintained?

8. How much genetic change accompanies speciation?

Rates of change within and between species.

9. Problems of classification: systematists (phenetics) vs. cladists (genealogical).

10. The puzzle of immunosystems. How do you account for antibody diversity?

11. Is every mutation and allelic substitution new? Genomic changes, types and frequency of mutation, turnover processes.

12. Phenotypes (expression) vs. genotypes (informational); homeostasis and canalization (large number of genotypes produce small number of phenotypes?). Linkage relationships and association on blocks of genes, developmental constraints, ontogenetic rules (the paradox of discrete classes of genotypes as against continuous variation in phenotypes). Can macroevolution be done on the phenotype level?

Do we need to know genotypes?

13. Are there optimization principles underlying evolutionary change? Is there direction to evolution, or are there many solutions? Are gene redundancies selected for? 
By contrast, the selectionists believe that with concomitant advances in molecular biology, the selective differences among allozymes will be uncovered. Preliminary distinctions have already been made between the abilities of variant enzyme forms to exploit variable vs. specific substrates. Some argue that "heterozygotes" produce enzyme forms that are better able to function over a wider range of temperatures, $\mathrm{pH}$ levels, cell conditions, etc. In the context of blocks of such genes, it is sometimes argued that polymorphism makes it possible for more than one gene (or allele) to be switched on at the same time or different times. In this perspective, regulation and development benefit from polymorphism. Suggestions on the pertinence of patterns of biochemical frequency-dependent selection are also put forth, e.g., by Clarke (1979).

Parallel to this vast unresolved allozyme variability, molecular biologists have discovered extensive repetitive (satellite) DNA segments whose purposes are not understood. Some claim that this satellite DNA has little, if any, function, others that its causality relationships constitute a challenging problem. It may be, for example, that these segments provide extra insurance for an essential regulatory, biosynthetic activity such that if a defect in one segment occurs, one of the duplicate sequences takes over and accomplishes the desired task. Alternatively, or in addition, they may provide material and opportunity for evolving new genetic ways of coping with new environmental conditions while maintaining the old systems still programmed. In this respect, repetitive DNA can be seen as performing like sex or recombination: i.e., it allows for easy access to sufficient DNA material that may be useful for responding to a changed environment. Evolution is an open dynamic system that maintains a balance between the needs of the present and the unknowns of the future. Such processes as recombination, diploidy, polyploidy, repetitive DNA and DNA transposition interact with and supplement the previously established machinery that is geared for the current environment.

5. Background for theoretical studies of sex ratio evolution. Sex ratio evolution is under intensive study from many perspectives (for recent books, see Charnov (1982), Bell (1982), Maynard Smith (1978), G. C. Williams (1975)). The study of nonlinear dynamic systems in population genetics-e.g., Karlin (1978), Lessard (1984), Karlin and Lessard (1983, 1984) - has made important progress toward describing the stable-equilibrium and dynamic properties of the general sex-differentiated viability model. A complete analysis is now possible for a broad class of sex ratio determination models. The "optimal" properties of an even $(1: 1)$ sex ratio have been established for a wide variety of models, extending previous works on the subject; these results will be elaborated in $\$ 7$. In $\$ 6$ we present previous and new pertinent mathematical analyses on the evolution of a panmictic (randomly mating) population subject to viability selection. $\$ 8$ discusses some sex-determination models governed by a continuous phenotypic variable.

The variety of sex-determining systems and controls is manifold. In broad terms these are distinguished by genotypic and environmental determinants subject to zygotic, parental, or population controls. Even complete genetic controls of sex expression can be manipulated by hormonal and physiological covariates. We provide Table 2 for added perspective. 
TABLE 2. Major Modes of Sex-Determining Systems and Controls

\begin{tabular}{|c|c|}
\hline Form of sex determination & Examples \\
\hline $\begin{array}{l}\text { I. Single gene } \\
\text { (multiple alleles) } \\
\text { and chromosomal } \\
\text { determinants }\end{array}$ & 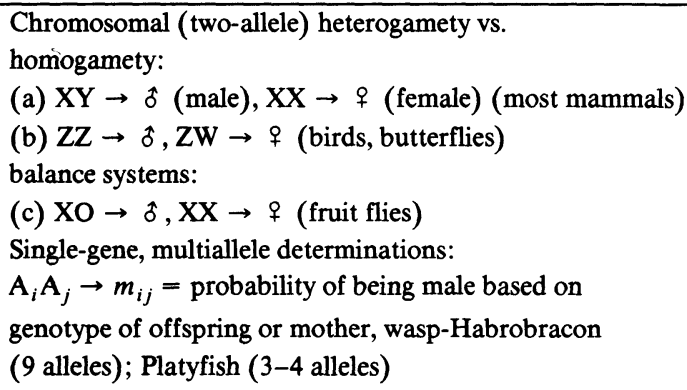 \\
\hline $\begin{array}{l}\text { II. Multifactorial } \\
\text { (many genes) }\end{array}$ & $\begin{array}{l}\text { In some fish (Poecelid) and plant populations } \\
\text { (e.g., melons and cucumbers) }\end{array}$ \\
\hline $\begin{array}{l}\text { III. Mixed parthenogenic and } \\
\text { bisexual reproduction } \\
\text { (haplodiploid) }\end{array}$ & $\begin{array}{l}\text { In most cases unfertilized eggs (haploids) develop } \\
\text { into males and fertilized eggs can be male or female. } \\
\text { Examples: ants, bees, wasps, mites. }\end{array}$ \\
\hline $\begin{array}{l}\text { IV. Hermaphroditism } \\
\text { (simultaneous, } \\
\text { sequential) }\end{array}$ & $\begin{array}{l}\text { Most plants produce both male and female sex gametes, } \\
\text { ova and pollen. Snails, earthworms, and tapeworms } \\
\text { are male and female at the same time. Certain } \\
\text { shrimp, and mollusks reproduce as male early in } \\
\text { life and later reverse to female. Other } \\
\text { fish do the opposite. }\end{array}$ \\
\hline V. Environmental responses & $\begin{array}{l}\text { Some reptiles (crocodiles, turtles) have } \\
\text { sex expression depending on temperature at incu- } \\
\text { bation; the sex of nematodes and copepods can be a } \\
\text { function of density of population. For parasi- } \\
\text { tic wasps, the sex of offspring can relate to } \\
\text { size and health of the host. }\end{array}$ \\
\hline $\begin{array}{l}\text { VI. Extrachromosomal and } \\
\text { cytoplasmic determination }\end{array}$ & $\begin{array}{l}\text { E.g., affected by viral particles, contagion, } \\
\text { meiotic drive }\end{array}$ \\
\hline VII. Incompatibility systems & $\begin{array}{l}\text { In some plant systems self-incompatible classes } \\
\text { (multisex types) }\end{array}$ \\
\hline
\end{tabular}

There are two principal approaches to the study of sex ratio evolution centering on (1) optimization and adaptive criteria, (2) sex-determining systems and controls.

The high incidence of equal male to female numbers at birth in mammal populations is striking compared to the frequent deviations from a $1: 1$ sex ratio in invertebrate species. G. C. Williams (1979) surveys several data sets of the literature reporting on litter sex ratio in mammals including deer, mouse, rabbit, mink, marmosets, humans, and a number of bird populations; see also Charnov (1982, Chapter 7). The evidence strongly supports a $1: 1$ litter sex ratio. Although Williams argues that this ratio is to be expected on the basis of straight Mendelian segregation, he offers no satisfactory account of how it evolved and what selective advantages it offers.

A mixed qualitative-quantitative argument predicting a $1: 1$ sex ratio is as follows. Consider the following three generations: I, grandparents; II, parents 
consisting of $n_{1}$ males and $n_{2}$ females who produce an aggregate of $N$ offspring in generation III. Then the average number of children is $N / n_{1}$ for a male of generation II, and $N / n_{2}$ for a female of generation II. Consider a typical grandparent of generation I who contributes a total of $m$ offspring comprised of $x$ males and $m-x$ females. The expected number of descendants for this grandparent in generation III is

$$
T=x \frac{N}{n_{1}}+(m-x) \frac{N}{n_{2}}=\frac{m N}{n_{2}}+\left(\frac{1}{n_{1}}-\frac{1}{n_{2}}\right) N x .
$$

Now $T$ is an increasing function of $x$ when $n_{2}>n_{1}$, and decreasing when $n_{2}<n_{1}$. Therefore the expected number of descendants of a grandparent of generation III increases if he (or she) produces more offspring of the presently rarer sex. Thus, when each grandparent imparts the same amount of genetic material (information) to its progeny the expected sex ratio should be $1: 1$. When males "cost" $\phi$ compared to 1 for females (akin to a viability differential factor for males versus females), the adjustment of even-sex ratio is $r^{*}=$ $\phi /(1+\phi)$, where $r^{*}$ is the value at which parental expenditure is equalized between the sexes for disparate costs in rearing (or producing) males and females.

The following model of Shaw and Mohler (1953) illustrates a kind of heuristic structural stability analysis in the context of sex ratio evolution (cf. Charnov (1982, Chapter 1)). Consider a dioecious population with $N+1$ females each producing $C^{\prime}$ offspring of which a proportion $r$ are male. Each offspring in turn produces a total of $K$ children (the grandchildren of the original females). Suppose there is a single deviant female $\tilde{F}$ with progeny sex ratio $\tilde{r}$. The expected number of genes passed by $\tilde{F}$ through grandsons is

$$
\frac{1}{2} K \frac{C \tilde{r}}{C \tilde{r}+N C r}
$$

(the $1 / 2$ factor occurs because she only contributes half the genes, her mate the other half), and through granddaughters

$$
\frac{1}{2} K\left\{\frac{C(1-\tilde{r})}{C(1-\tilde{r})+N C(1-r)}\right\} .
$$

If $N$ is large, the total fitness of $\tilde{F}$ assessed as the cumulative number of her genes transferred to the third generation is approximately

$$
\frac{1}{2} \frac{K}{N}\left(\frac{\tilde{r}}{r}+\frac{1-\tilde{r}}{1-r}\right)
$$

compared to $\frac{1}{2}(K / N)$ for a typical female.

Elementary analysis of (5.1) shows that for $r \neq 1 / 2$ there exists $\tilde{r}$ (closer to $1 / 2$ ) which gives a greater contribution of genes to the subsequent generation compared to the genetic output of $r$. But for $r=1 / 2$ no $\tilde{r} \neq 1 / 2$ can secure a larger (reproductive) fitness. This attribute of $r=1 / 2$ is called ESS (evolutionary stable strategy); compare this to Theorem 7.5 below.

In the next three sections we set forth a more thorough study of mathematical models of balances in differential viability effects and sex ratio evolution in randomly mating populations. 
6. Bisexual multiallele viability selection systems. Our objective in $\$ \S 6$ and 7 is to formulate and analyze a general class of mathematical models pertainng to sex ratio evolution. The associated dynamical system is inherently related to the study of bisexual multiallelic viability selection.

Consider a diploid sex-differentiated population characterized by $r$ alleles $A_{1}, \ldots, A_{r}$ at an autosomal locus with associated genotypes $A_{i} A_{j}$. Under the effects of differential viability selection, random mating, and Mendelian segregation, the parameters of the model are as follows. The viability fitness matrix for females is $F=\left\|f_{i j}\right\|_{1}^{r}$ and for males $M=\left\|m_{i j}\right\|_{1}^{r}$, respectively, where the quantity $f_{i j}\left(m_{i j}\right)$ is interpreted as the relative number of $\mathbf{A}_{i} \mathbf{A}_{j}$ female (male) zygotes that survive to contribute to the next generation. The frequency of genotype $\mathrm{A}_{i} \mathrm{~A}_{j}$ in the female population is denoted by $2 p_{i j}$ when $i \neq j$, and $p_{i i}$ for $i=j$. Accordingly, the frequency of allele $\mathrm{A}_{i}$ in this population is $p_{i}=$ $\sum_{j=1}^{r} p_{i j}$. The corresponding frequencies for the male population are denoted by $2 q_{i j}, q_{i i}$, and $q_{i}$.

Under viability selection the results from random mating are equivalent to random union of gametes (e.g., see $\operatorname{Karlin}(1978, \$ 1)$ ) and therefore the collection of all $\mathrm{A}_{i} \mathrm{~A}_{j}$ zygotes is $p_{i} q_{j}+q_{i} p_{j}$. Taking account of differential viability selection and Mendelian segregation for a $1: 1$ sex ratio, the genotypic frequencies over two successive generations obey the recursion relations

$$
q_{i j}^{\prime}=\frac{m_{i j}\left(p_{i} q_{j}+q_{i} p_{j}\right)}{2 w}, \quad p_{i j}^{\prime}=\frac{f_{i j}\left(p_{i} q_{j}+q_{i} p_{j}\right)}{2 v},
$$

where

$$
w=w(\mathbf{p}, \mathbf{q})=\sum_{i, j} m_{i j} p_{i} q_{j} \quad \text { and } \quad v=\sum_{i, j} f_{i j} p_{i} q_{j} .
$$

The allele frequencies $p_{i}^{\prime}$ and $q_{i}^{\prime}$ of the next generation are calculated from the transformation equations

$$
\begin{gathered}
p_{i}^{\prime}=\frac{\frac{1}{2}\left[p_{i}\left(\sum_{j=1}^{r} f_{i j} q_{j}\right)+q_{i}\left(\sum_{j=1}^{r} f_{i j} p_{j}\right)\right]}{\sum_{i, j=1}^{r} f_{i j} p_{i} q_{j}}, \quad i=1,2, \ldots, r . \\
q_{i}^{\prime}=\frac{\frac{1}{2}\left[p_{i} \sum_{j=1}^{r} m_{i j} q_{j}+q_{i} \sum_{j=1}^{r} m_{i j} p_{j}\right]}{\sum_{i, j=1}^{r} m_{i j} p_{i} q_{j}}, \quad i=1,2, \ldots, r .
\end{gathered}
$$

It will be convenient to employ vector notation. To this end the Schur product of the vectors $\mathbf{a}=\left(a_{1}, \ldots, a_{r}\right)$ and $\mathbf{b}=\left(b_{1}, \ldots, b_{r}\right)$ is $\mathbf{a} \circ \mathbf{b}=$ $\left(a_{1} b_{1}, a_{2} b_{2}, \ldots, a_{r} b_{r}\right)$. The Euclidean inner product of two vectors is denoted by $\langle\mathbf{a}, \mathbf{b}\rangle=\sum_{i=1}^{r} a_{i} b_{i}$.

In the foregoing notation the recursion relations (6.2) take the form

$$
\mathbf{p}^{\prime}=\frac{1}{2} \frac{\mathbf{p} \circ F \mathbf{q}+\mathbf{q} \circ F \mathbf{p}}{\langle\mathbf{p}, F \mathbf{q}\rangle}, \quad \mathbf{q}^{\prime}=\frac{1}{2} \frac{\mathbf{q}^{\circ} M \mathbf{p}+\mathbf{p} \circ M \mathbf{q}}{\langle\mathbf{p}, M \mathbf{q}\rangle} .
$$

The designation $\mathbf{p} \circ F$ stands for the matrix product $\mathbf{D}_{\mathbf{p}} F$ where $D_{\mathbf{p}}$ is the diagonal matrix having the components of $\mathbf{p}$ down the main diagonal. Relations (6.3) present a nonlinear transformation $T$ of $2 r$ variables $((2 r-2)$ independent ones, since $\left.\sum_{i=1}^{r} p_{i}=\sum_{i=1}^{r} q_{i}=1\right)$. 
In the case $M=F$ (such that selection operates on the male and female zygotes in the same manner), then $\mathbf{p}^{\prime}=\mathbf{q}^{\prime}$, and in all subsequent generations the transformation $T$ can be reduced to the single set

$$
\mathbf{p}^{\prime}=\frac{\mathbf{p} \circ M \mathbf{p}}{\langle\mathbf{p}, M \mathbf{p}\rangle}=S \mathbf{p}
$$

where the sexes need not be distinguished. Let the simplex of frequency vectors $\mathbf{p}=\left(p_{1}, p_{2}, \ldots, p_{r}\right), p_{i} \geqslant 0, \Sigma p_{i}=1$ be denoted by $\Delta$, its interior by $\Delta^{0}$.

The symmetric multiallele viability model. The analysis of the transformation (6.4), its convergence and equilibrium properties, has received much attention (e.g., Kingman (1961a,b), Crow and Kimura (1970), Ewens (1979), Karlin (1978, and references therein)). We review several facts concerning (6.4) needed for our later discussion of (6.2).

The mean fitness function

$$
w(\mathbf{p})=\sum_{i, j=1}^{r} m_{i j} p_{i} p_{j}=\langle M \mathbf{p}, \mathbf{p}\rangle
$$

provides a strict Lyapounov function for the mapping (6.4) such that

$$
w(S \mathbf{p}) \geqslant w(\mathbf{p}) \quad \text { with equality if and only if } \mathbf{p}^{\prime}=S \mathbf{p}=\mathbf{p} .
$$

This remarkable property is sometimes referred to as the discrete form of the fundamental theorem of natural selection (Crow and Kimura (1970, Chapter 5), Ewens (1979, Chapter 2)).

Inequality (6.6) reduces to

$$
\sum_{i, j} m_{i j} p_{i} p_{j} w_{i} w_{j} \geqslant[w(\mathbf{p})]^{3}
$$

where $w_{i}=\sum_{j=1}^{r} m_{i j} p_{j}, i=1,2, \ldots, r$.

An elegant proof of (6.7) appears in Kingman (1961a); see (6.10) below. A wider perspective derives inequality (6.7) as a special case of the generalized moment inequality

$$
\left(\frac{\langle C \mathbf{x}, \mathbf{x}\rangle}{\langle\mathbf{x}, \mathbf{x}\rangle}\right)^{k} \leqslant \frac{\left\langle C^{k} \mathbf{x}, \mathbf{x}\right\rangle}{\langle\mathbf{x}, \mathbf{x}\rangle}
$$

where $C=\left\|c_{i j}\right\|_{1}^{r}$ is a symmetric matrix of positive elements, $\mathbf{x}=\left(x_{1}, x_{2}, \ldots, x_{r}\right)$ a positive vector and $k$ a positive integer.

Equality holds in (6.8) if and only if $\mathbf{x} \in \Delta$ is a principal eigenvector $C \mathbf{x}=\rho(C) \mathbf{x}$ for $\rho(C)$ equal to the spectral radius of $C$. (The result of (6.8) is proved by induction with respect to the dimension $r$.) The subtlety of (6.8) (for $k$ odd) resides in the fact that $C$, although symmetric with positive elements, is not necessarily positive definite.

We show how to deduce (6.7) from (6.8) with $k=3$. Indeed for any frequency vector $\mathbf{p} \in \Delta$ let $C=D_{\sqrt{p}} M D_{\sqrt{p}}$, where $D_{\mathbf{y}}$ is the diagonal matrix with the components $\mathbf{y}=\left(y_{1}, \ldots, y_{r}\right)$ down the diagonal (we use the notation 
$\left.\sqrt{\mathbf{p}}=\left(\sqrt{p_{1}}, \sqrt{p_{2}}, \ldots, \sqrt{p_{r}}\right)\right)$. The left-hand side of $(6.7)$ is $($ since $\langle\sqrt{\mathbf{p}}, \sqrt{\mathbf{p}}\rangle=$ $\left.\sum p_{i}=1\right)$

$$
\begin{aligned}
\langle M(\mathbf{p} \circ M \mathbf{p}), \mathbf{p} \circ M \mathbf{p}\rangle & =\left\langle C^{3} \sqrt{\mathbf{p}}, \sqrt{\mathbf{p}}\right\rangle=\left\langle C^{3} \sqrt{\mathbf{p}}, \sqrt{\mathbf{p}}\right\rangle /\langle\sqrt{\mathbf{p}}, \sqrt{\mathbf{p}}\rangle \\
& \geqslant(\langle C \sqrt{\mathbf{p}}, \sqrt{\mathbf{p}}\rangle)^{3}=\langle M \mathbf{p}, \mathbf{p}\rangle^{3},
\end{aligned}
$$

the last inequality resulting because of (6.8). Equality holds if and only if $C \sqrt{\mathbf{p}}=\rho(C) \sqrt{\mathbf{p}}$, which is equivalent to $\sqrt{\mathbf{p}} M \mathbf{p}=\sqrt{\mathbf{p}} w(\mathbf{p})$ or $\sqrt{\mathbf{p}} \circ(M \mathbf{p}-$ $\mathbf{u w}(\mathbf{p}))=0(\mathbf{u}=(1, \ldots, 1)$ is the vector of all unit components). The latter equality means that $p$ is an equilibrium point of the transformation $S$ of (6.4).

For other purposes we record Kingman's (1961a) proof of (6.7):

$$
\begin{aligned}
& \sum_{i, j} m_{i j} p_{i} w_{i} p_{j} w_{j}=\sum_{i, j, k} m_{i j} m_{i k} p_{i} p_{j} p_{k} w_{j}=\sum_{i, j, k} m_{i j} m_{i k} p_{i} p_{j} p_{k}\left(\frac{w_{j}+w_{k}}{2}\right) \\
& \geqslant \sum_{i, j, k} m_{i j} m_{i k} p_{i} p_{j} p_{k} \sqrt{w_{j}} \sqrt{w_{k}}=\sum_{i} p_{i}\left(\sum_{j} m_{i j} p_{j} \sqrt{w_{j}}\right)^{2} \geqslant\left(\sum_{i, j} p_{i} m_{i j} p_{j} \sqrt{w_{j}}\right)^{2} \\
& =\left(\sum_{j} p_{j} w_{j}^{3 / 2}\right)^{2} \geqslant\left(\sum_{j} p_{j} w_{j}\right)^{3}=w^{3}
\end{aligned}
$$

the last inequality coming by Holder's inequality.

For $0 \leqslant m_{i j} \leqslant 1$, we can improve on the inequality of (6.7). First, we show

$$
0 \leqslant \sum_{i, j} m_{i j} p_{i} p_{j} w_{i} w_{j}-w^{3} \leqslant 2\left[\sum_{i} p_{i} w_{i}^{2}-w^{2}\right]
$$

for $w=w(\mathbf{p})$.

Indeed, we start with the identity

$$
\begin{aligned}
\sum_{i, j}\left(1-m_{i j}\right) p_{i} p_{j}\left(1-w_{i}\right) & \left(1-w_{j}\right)-(1-w)^{3} \\
= & 2\left[\sum_{i} p_{i} w_{i}^{2}-w^{2}\right]-\sum_{i, j} m_{i j} p_{i} p_{j} w_{i} w_{j}+w^{3} .
\end{aligned}
$$

By virtue of (6.7), the left side is nonnegative and then (6.11) follows.

Instead of the last step in (6.10), by using Jensen's inequality for a random variable $X=w_{j}$ with probability $p_{j}$, and so $E[X]=\sum w_{j} p_{j}=w$ ( $E$ for expectation), we have

$$
E[f(X)] \geqslant f[E(X)]+(B / 2) \sigma^{2}(X) \text { for } f(\xi)=\xi^{3 / 2},
$$

where

$$
\sigma^{2}(X)=\operatorname{Var} X=\sum_{i} p_{i} w_{i}^{2}-w^{2}=\sum_{i} p_{i}\left(w_{i}-w\right)^{2}=\sigma^{2}
$$

and $B=3 / 4$ is a lower bound of $f^{\prime \prime}(\xi)$ on $0 \leqslant \xi \leqslant 1$. It follows when all $w_{j} \leqslant 1$ that (Lyubich et al. (1980))

$$
\sum_{i, j} m_{i j} p_{i} p_{j} w_{i} w_{j} \geqslant\left(\sum p_{j} w_{j}^{3 / 2}\right)^{2} \geqslant\left(w^{3 / 2}+\frac{3}{8} \sigma^{2}\right)^{2} \geqslant w^{3}+\frac{3}{4} \sigma^{2} w^{2} .
$$


Employing this inequality on the left in (6.12) with some further manipulations, we get

$$
\begin{aligned}
\frac{3}{4} w^{2} \sigma^{2} & =\frac{3}{4} w^{2}\left[\sum_{i} p_{i}\left(w_{i}-w\right)^{2}\right] \leqslant \sum_{i, j} m_{i j} p_{i} p_{j} w_{i} w_{j}-w^{3} \\
& \leqslant\left[2-\frac{3}{4}(1-w)^{2}\right]\left[\sum_{i} p_{i}\left(w_{i}-w\right)^{2}\right]=\left[2-\frac{3}{4}(1-w)^{2}\right] \sigma^{2}
\end{aligned}
$$

(In Appendix A to this section we develop a number of information theoretic inequalities connected with the transformation (6.4) akin to (6.7).)

To avoid unessential technical details we assume, henceforth, unless stated otherwise, that $M$ satisfies the following genericy conditions.

DEFINITION 6.1. The matrix $M$ is said to be generic if:

(a) $0<m_{i j}<1$ for all $i$ and $j$;

(b) every principal submatrix of $M$ is nonsingular;

(c) for any submatrix of indices $i_{1}<i_{2}<\cdots<i_{k}$ the solution $\left\{\gamma_{\nu}\right\}$ of

$$
\sum_{\nu=1}^{k} m_{i_{\mu}, i_{\nu}} \gamma_{\nu}=1, \quad \mu=1,2, \ldots, k,
$$

has

$$
\sum_{\nu=1}^{k} m_{i_{0}, i_{\nu}} \gamma_{\nu} \neq 1 \text { for all } i_{0} \neq i_{\mu}
$$

These requirements guarantee that every equilibrium state of $S \mathbf{p}=\mathbf{p}, \mathbf{p} \in \Delta$ is an isolated fixed point whose stability can be decided by a local linear approximation. Unless explicitly stated otherwise we assume Definition 6.1 in force.

A polymorphic equilibrium $\mathbf{p}^{*}$ (i.e., an internal equilibrium) of system (6.4) exists if the linear system of equations

$$
\sum_{j=1}^{r} m_{i j} \xi_{j}=1, \quad i=1,2, \ldots, r
$$

admits a positive solution and then $p_{i}^{*}=\xi_{i} / \sum_{j=1}^{r} \xi_{j}$. This polymorphic equilibrium is locally stable if any of the following conditions holds (Kingman (1961b)):

(i) The eigenvalues of $M$ satisfy

$$
\lambda_{1}(M)>0>\lambda_{2}(M) \geqslant \cdots \geqslant \lambda_{r}(M) .
$$

(ii) Relations (i) also obtain if the successive principal determinants $D_{k}=$ $\operatorname{det}\left\|m_{i j}\right\|_{1}^{k}$ strictly alternate in sign.

An important fact in one-locus multiallele selection theory is that a locally stable polymorphic equilibrium is globally stable relative to all states $\mathbf{p} \in \Delta^{0}$.

With any number of alleles $s \leqslant r$, an equilibrium vector $\hat{\mathbf{p}}=\left(\hat{p}_{1}, \ldots, \hat{p}_{s}\right)$ solves the system of equations

$$
\hat{w}_{j}=(M \hat{\mathbf{p}})_{j}=\sum_{j=1}^{r} m_{i j} \hat{p}_{j}=w(\hat{\mathbf{p}})=\langle\hat{\mathbf{p}}, M \hat{\mathbf{p}}\rangle, \quad i \in l(\hat{\mathbf{p}}),
$$


the equations applying for those indices $l(\hat{\mathbf{p}})$ where $\hat{p}_{i}>0$. The equilibrium $\hat{\mathbf{p}}$ is internally stable provided the reduced matrix $\hat{M}$ of $M$ restricted to the rows and columns of $l(\hat{\mathbf{p}})$ satisfies (6.15).

External stability of a boundary equilibrium prevails if any new allele, introduced in small frequency, is ultimately eliminated under iteration of the transformation $S$ of (6.4). This is equivalent to the condition

$$
w(\hat{\mathbf{p}})>(M \hat{\mathbf{p}})_{k} \text { applies for all } k \text { where } \hat{p}_{k}=0 \text {, i.e., for } k \notin l(\hat{\mathbf{p}}) \text {. }
$$

The attributes (6.15), $\hat{\mathbf{p}} \in \Delta^{0}$ and (6.14) can apply together yielding a globally stable polymorphism or (6.14) without (6.15) presenting an unstable polymorphism or (6.15) without (6.14). In the latter event we have proved that a unique stable equilibrium (possibly on the boundary of $\Delta$ ) exists.

The fact that $w(\mathbf{p})$ is a strict Lyapounov function for the transformation (6.4) leads to the standard result.

Proposition 6.1. The iterates $S^{n} \mathbf{p}$ converge for every $\mathbf{p} \in \Delta$. Let $\hat{\mathbf{p}}$ be an equilibrium ( $S \hat{\mathbf{p}}=\hat{\mathbf{p}})$. If $\boldsymbol{w}(\hat{\mathbf{p}})$ is a strict local maximum of $w(\mathbf{p})$ (relative to $\Delta)$, then $\hat{\mathbf{p}}$ is a locally stable equilibrium for the transformation $S$ of (6.4).

Actually global convergence can be proved without the need of the genericy Definition 6.1 (Lyubich et al. (1980)).

Proposition 6.2 (Kingman (1961b)). An interior fixed point $\mathbf{p}^{*}$ is locally stable if and only if

$$
\langle M \boldsymbol{\xi}, \boldsymbol{\xi}\rangle<0 \text { for all } \boldsymbol{\xi} \equiv \mathbf{0} \text { satisfying }\langle\boldsymbol{\xi}, \mathbf{u}\rangle=0,
$$

i.e., $M$ is negative definite on the linear manifold orthogonal to $\mathbf{u}: \mathscr{L}_{\mathbf{u}}=\{\boldsymbol{\xi} \mid\langle\boldsymbol{\xi}, \mathbf{u}\rangle$ $=0\}$. Moreover, $\mathbf{p}^{*}$ is locally stable if and only if $\mathbf{p}^{*}$ is globally stable in that $S^{n} \mathbf{p} \rightarrow \mathbf{p}^{*}$ for every $\mathbf{p} \in \Delta^{0}$, and

(6.18b) $w\left(\mathbf{p}^{*}\right)$ is a strict maximum of $w(\mathbf{p})$ with respect to all $\mathbf{p} \in \Delta$.

Consider the gradient map $S^{\prime}(\mathbf{p})$ (i.e., the local linear approximation to $S$ at p). We find for $\mathbf{p}$ interior to $\Delta$,

$$
S^{\prime}(\mathbf{p}) \xi=\frac{\xi \circ M \mathbf{p}}{w(\mathbf{p})}+\frac{\mathbf{p} \circ M \xi}{w(\mathbf{p})}-\frac{2\langle M \mathbf{p}, \boldsymbol{\xi}\rangle}{[w(\mathbf{p})]^{2}} \mathbf{p} \circ M \mathbf{p} .
$$

When $\mathrm{p}^{*}$ is an interior fixed point of $S$ then (6.19) reduces to

$$
S^{\prime}\left(\mathbf{p}^{*}\right) \xi=\xi+\left(\mathbf{p}^{*} \circ M \xi\right) / w\left(\mathbf{p}^{*}\right)=\left(I+M^{*}\right) \xi
$$

with $\boldsymbol{\xi}$ restricted to satisfy $\langle\boldsymbol{\xi}, \mathbf{u}\rangle=0, \mathbf{u}=(1,1, \ldots, 1)$. The eigenvalues of $S^{\prime}\left(\mathbf{p}^{*}\right)$ are $1+\gamma_{i}, i=1,2, \ldots, r$, where $\gamma_{i}$ are the eigenvalues of the matrix $M^{*}=\mathbf{p}^{*} \circ M / w\left(\mathbf{p}^{*}\right)$. Moreover, $\gamma_{1}>\left|\gamma_{i}\right|, i=2, \ldots, r$, owing to the Frobenius theorem on positive matrices. Since $\mathbf{u} M^{*}=\mathbf{u}$, it follows that $\gamma_{1}=1$. Global stability (with respect to $\Delta^{0}$ ) is guaranteed if $\left|1+\gamma_{i}\right|<1$ or $\gamma_{i}<0$ for $i \geqslant 2$.

Appendix B describes some ancillary consequences of the internal stability conditions (6.18a) of independent interest.

We next describe a new characterization for equilibrium states and stability for the dynamical system (6.4) that will play an important role in our analysis of the dynamical equations underlying sex ratio evolution expounded in $§ 7$. 
For each $\mathbf{p}$ in $\Delta^{0}$ we form the matrix

$$
B(\mathbf{p})=D_{\mathbf{p}} M+D_{M \mathbf{p}} \quad\left(D_{\mathbf{p}}\right. \text { is the diagonal matrix with the }
$$

components of $\mathbf{p}$ down the main diagonal).

If the matrix $M$ (and therefore $B(p)$ ) is positive, we know by the PerronFrobenius theory that the eigenvalue of largest magnitude for $B(p)$ is positive, admitting a unique (apart from a scale factor) strictly positive right eigenvector $\mathbf{y}>\mathbf{0}$. We denote by $\rho(\mathbf{p})$ the spectral radius of the matrix $B(\mathbf{p})$ and take the associated positive eigenvector $\mathbf{y}$ satisfying $\sum_{i=1}^{r} y_{i}=\langle\mathbf{y}, \mathbf{u}\rangle=1$. Then $\mathbf{y}=\mathbf{y}(\mathbf{p})$ and $\rho(p)$ vary continuously (actually analytically) as a function of $\mathbf{p}$ in $\Delta^{0}$. The principal eigenvalue-eigenvector identity is

$$
B(\mathbf{p}) \mathbf{y}(\mathbf{p})=\rho(\mathbf{p}) \mathbf{y}(\mathbf{p}) .
$$
$\Delta$.

The spectral function $\rho(p)$ can be extended by continuity to the boundary of

THEOREM 6.1 (KARLIN AND LESSARD (1983)). Let $M$ be a positive symmetric matrix and generic in the sense of Definition 6.1. Let $\rho(\mathbf{p})$ be the spectral radius of the matrix $B(\mathbf{p})$ for $\mathbf{p} \in \Delta^{0}$.

(i) $\mathbf{p}^{*}$ is a polymorphic equilibrium of (6.4) if and only if $\mathbf{p}^{*}$ is a critical point of the function $\rho(\mathbf{p})$, meaning that the derivative of $\rho(\mathbf{p})$ in every direction at $\mathbf{p}^{*}$ relative to the simplex $\Delta^{0}$ is zero.

(ii) $\mathbf{p}^{*}$ is a stable polymorphic equilibrium if and only if $\rho(\mathbf{p})$ achieves a strict local maximum with respect to $\Delta$ at $\mathbf{p}^{*}$.

For a boundary equilibrium frequency vector, the corresponding result is

TheOREM 6.2 (KARLIN AND LeSSARD (1983)). Let $M$ be generic (in the sense of Definition 6.1).

(i) $A$ boundary equilibrium $\hat{\mathbf{p}}$ of $S \mathbf{p}=\mathbf{p}$ is stable if and only if $\rho(\hat{\mathbf{p}})$ is a strict local maximum of $\rho(\mathbf{p})$ with respect to $\mathbf{p}$ in $\Delta$.

(ii) The local maxima of $\rho(\mathbf{p})$ and the mean fitness function $w(\mathbf{p})=\sum_{i, j} m_{i j} p_{i} p_{j}$ are achieved at the same points which are the stable equilibria of the transformation (6.4). An interior local maximum is actually a global maximum.

The proof of Theorem 6.1 is given in Appendix $\mathrm{C}$ to this section.

The two-sex viability model. For the general sex-differentiated model (6.3) (allowing $M \neq F$ ) there can exist multiple interior equilibria in $\Delta \otimes \Delta$ and, therefore, any associated Lyapounov function cannot be a multivariate quadratic polynomial or a ratio of quadratic forms. Numerical studies persistently exhibit global convergence of the iterates of (6.3). Nevertheless, the ascertainment of a Lyapounov function for the general sex-differentiated viability model remains an open problem.

The case of

$$
F=a U+b M
$$

(where $U$ is the $r \times r$ matrix of all unit entries, $a, b$ are constants) in (6.3) is of special interest since any equilibrium $\mathbf{p}^{*}$ of (6.4) engenders an equilibrium pair for equations (6.3),

$$
\{\hat{\mathbf{q}}, \hat{\mathbf{p}}\}=\left\{\mathbf{p}^{*}, \mathbf{p}^{*}\right\}
$$


referred to henceforth as a symmetric (in the sexes) equilibrium. There may also exist (and often do) nonsymmetric equilibrium combinations $\{\tilde{\mathbf{q}}, \tilde{\mathbf{p}}\}$ with $\tilde{\mathbf{q}} \neq \tilde{\mathbf{p}}$.

Let $\{\hat{\boldsymbol{p}}, \hat{\mathbf{q}}\}$ be a polymorphic fixed point of (6.3), so that $\hat{p}_{i} \hat{q}_{j}>0$ for all $i, j$. The gradient matrix at $\{\hat{\boldsymbol{p}}, \hat{\mathbf{q}}\}$ can be standardly determined to obtain, for the corresponding gradient matrix mapping,

$$
\begin{aligned}
\xi^{\prime} & =C_{F, \hat{\mathbf{q}}} \boldsymbol{\xi}-\frac{\hat{\mathbf{p}}\langle\boldsymbol{\xi}, F \hat{\mathbf{q}}\rangle}{\langle\hat{\mathbf{p}}, F \hat{\mathbf{q}}\rangle}+C_{F, \mathbf{p} \eta}-\frac{\hat{\mathbf{p}}\langle\boldsymbol{\eta}, F \hat{\mathbf{p}}\rangle}{\langle\hat{\mathbf{p}}, F \hat{\mathbf{q}}\rangle}, \\
\eta^{\prime} & =C_{M, \hat{\mathbf{q}}} \boldsymbol{\xi}-\frac{\hat{\mathbf{q}}\langle\boldsymbol{\xi}, M \hat{\mathbf{q}}\rangle}{\langle\hat{\mathbf{p}}, M \hat{\mathbf{q}}\rangle}+C_{M, \mathbf{p} \eta}-\frac{\hat{\mathbf{q}}\langle\boldsymbol{\xi}, M \hat{\mathbf{p}}\rangle}{\langle\hat{\mathbf{p}}, M \hat{\mathbf{q}}\rangle},
\end{aligned}
$$

where

$$
C_{F, \hat{q}} \xi=\frac{1}{2} \frac{\xi \circ F \hat{\mathbf{q}}+\hat{\mathbf{q}} \circ F \boldsymbol{\xi}}{\langle\hat{\mathbf{p}}, F \hat{\mathbf{q}}\rangle},
$$

with the matrix transformations $C_{F, \hat{\mathrm{p}}}, C_{M, \hat{\mathrm{q}}}, C_{M, \hat{\mathrm{p}}}$ determined analogously. We can write the linear approximation of $T$ in (6.3) near $\{\hat{\mathbf{p}}, \hat{\mathbf{q}}\}$ as

$$
\left(\begin{array}{c}
\boldsymbol{\xi}^{\prime} \\
\eta^{\prime}
\end{array}\right)=\left(\begin{array}{ll}
P_{1} & P_{2} \\
Q_{1} & Q_{2}
\end{array}\right)\left(\begin{array}{l}
\boldsymbol{\xi} \\
\eta
\end{array}\right)
$$

where $P_{i}$ and $Q_{i}$ are appropriate $r \times r$ matrices depending on $\{\hat{\mathbf{p}}, \hat{\mathbf{q}}\}$ and the viability matrices $F$ and $M$ (e.g., $P_{1} \xi=C_{F, \hat{q}} \xi-\langle\xi, F \hat{\mathbf{q}}\rangle \hat{\mathbf{p}} /\langle\hat{\mathbf{p}}, F \hat{\mathbf{q}}\rangle$ ). The equilibrium $\{\hat{\boldsymbol{p}}, \hat{\mathbf{q}}\}$ is locally stable if the magnitudes of the eigenvalues for the transformation (6.27) on the invariant subspace $\mathscr{M}=\{\boldsymbol{\xi}, \boldsymbol{\eta}:\langle\boldsymbol{\xi}, \mathbf{u}\rangle=\langle\boldsymbol{\eta}, \mathbf{u}\rangle=$ $0\}$ are less than unity. It is useful to record some facts concerning (6.27) in the special case $P_{1}=P_{2}$ and $Q_{1}=Q_{2}$ such that the gradient matrix transformation has the form

$$
\left(\begin{array}{l}
\boldsymbol{\xi}^{\prime} \\
\boldsymbol{\eta}^{\prime}
\end{array}\right)=\left(\begin{array}{ll}
P & P \\
Q & Q
\end{array}\right)\left(\begin{array}{l}
\boldsymbol{\xi} \\
\boldsymbol{\eta}
\end{array}\right)=\Gamma\left(\begin{array}{l}
\boldsymbol{\xi} \\
\boldsymbol{\eta}
\end{array}\right)
$$

The following lemma is known and elementary.

LEMMA 6.1. Consider a matrix mapping of the form (6.28), with $\xi$ and $\eta$ in $E^{r}$. The eigenvalues of $\Gamma$ are those of $R=P+Q$ plus the eigenvalue 0 which occurs with additional multiplicity $r$.

EXAMPLE I. Consider viability matrices satisfying the relation $M=a U+b F$, $a, b \geqslant 0$, with general $F$, where $U=\left\|u_{i j}\right\|_{1}^{r}, u_{i j}=1$, for all $i, j$.

If there exists $\mathbf{p}^{*}$ such that $F \mathbf{p}^{*}=\gamma \mathbf{u}, \mathbf{p}^{*}$ a positive vector and $\gamma>0$, then $\left\{\mathbf{p}^{*}, \mathbf{p}^{*}\right\}$ is a polymorphic equilibrium for the bisexual transformation associated with $F$ and $M=a U+b F$, and the gradient matrix $T^{\prime}\left\{\mathbf{p}^{*}, \mathbf{p}^{*}\right\}$ has the form (6.28) with

$$
P \xi=\frac{1}{2} \xi+\frac{1}{2} \frac{\mathbf{p}^{*} \circ F \xi}{w_{F}\left(\mathbf{p}^{*}\right)} \quad \text { and } \quad Q \xi=\frac{1}{2} \xi+\frac{1}{2} \frac{\mathbf{p}^{*} \circ M \xi}{w_{M}\left(\mathbf{p}^{*}\right)} .
$$

Further, $w_{M}\left(\mathbf{p}^{*}\right)=a+b w_{F}\left(\mathbf{p}^{*}\right)$. 
(we use the notation $\left.\mathbf{x} / \mathbf{y}=\left(x_{1} / y_{1}, x_{2} / y_{2}, \ldots, x_{r} / y_{r}\right)\right)$ and note that

$$
M(\mathbf{p}) \mathbf{p}=\mathbf{p} \text {. }
$$

Since for $\mathbf{p} \in \Delta^{0}, M(\mathbf{p})$ is a positive matrix, we can conclude that 1 is the spectral radius of $M(\mathbf{p})$. Because $M(\mathbf{p})$ is symmetrizable (differs from a symmetric matrix by a multiplicative positive diagonal matrix) it follows that $I+M(\mathbf{p})$ is invertible and also $D_{M \mathbf{p}}(I+M(\mathbf{p}))=B(\mathbf{p})$ is invertible.

Recall the fact that if $M$ is a symmetric matrix and $D$ a positive diagonal matrix, then $\tilde{M}=D M$ acts as a symmetric matrix with respect to the modified inner product

$$
\langle\mathbf{x}, \mathbf{y}\rangle_{\mathbf{d}}=\sum_{i} \frac{1}{d_{i}} x_{i} y_{i}=\left\langle\frac{\mathbf{x}}{\mathbf{d}}, \mathbf{y}\right\rangle
$$

where $d_{i}$ are the diagonal elements of $D$.

We further note, when $\mathbf{p}^{*}$ is a polymorphism of $M$, since $\mathbf{u}$ is a simple left eigenvector of eigenvalue 1 for $M\left(\mathrm{p}^{*}\right)$, that $I+M\left(\mathrm{p}^{*}\right)$ (and also $\left(I-M\left(\mathrm{p}^{*}\right)\right)$ ) is invertible on $\mathscr{L}=\{\mathbf{z} ;\langle\mathbf{u}, \mathbf{z}\rangle=0\}$ onto itself. Finally, we record

LEMMA C.1. The frequency vector $\mathbf{p} \in \Delta^{0}$ is a polymorphic equilibrium of $S$ in (6.4) if and only if $B\left(\mathbf{p}^{*}\right) \mathbf{p}^{*}=\lambda^{*} \mathbf{p}^{*}$ and then $\lambda^{*}=2 w^{*}, w^{*}=\left\langle M \mathbf{p}^{*}, \mathbf{p}^{*}\right\rangle$, i.e., $\rho\left(\mathbf{p}^{*}\right)=2 w\left(\mathbf{p}^{*}\right)$.

Proof. If $\mathbf{p}^{*}$ is a polymorphism we have $M \mathbf{p}^{*}=w^{*} \mathbf{u}$. Then $M^{*} \mathbf{p}^{*}=$ $\left(\mathbf{p}^{*} \circ M \mathbf{p}^{*}\right) / w\left(\mathbf{p}^{*}\right)=\mathbf{p}^{*}$ and, also, $B\left(\mathbf{p}^{*}\right) \mathbf{p}^{*}=2 w^{*} \mathbf{p}^{*}$. Conversely, suppose $B\left(\mathbf{p}^{*}\right) \mathbf{p}^{*}=\lambda^{*} \mathbf{p}^{*}$ for $\mathbf{p}^{*} \in \Delta^{0}$. This requires that $\mathbf{p}^{*} \circ M \mathbf{p}^{*}=\lambda^{*} \mathbf{p}^{*} / 2$, and since $p_{i}^{*}>0$ for all indices, it follows that $M \mathbf{p}^{*}=\lambda^{*} \mathbf{u} / 2$, implying $\mathbf{p}^{*}$ is a polymorphism with $\lambda^{*}=2 w^{*}$.

Proof of Theorem 6.1. We first develop some preliminaries. Let $\mathbf{p}^{*} \in \Delta^{0}$. Choose a direction $\boldsymbol{\eta}=\left(\eta_{1}, \eta_{2}, \ldots, \eta_{r}\right)$ in $\Delta^{0}$, that is, $\boldsymbol{\eta}$ satisfying $\langle\boldsymbol{\eta}, \mathbf{u}\rangle=0$ and $s$ small enough obeying $\mathbf{p}(s)=\mathbf{p}^{*}+s \eta \in \Delta^{0}$. The Frobenius theory of positive matrices affirms that the spectral radius $\rho(s)=\rho(\mathbf{p}(s))$ of $B(s)=$ $B(\mathrm{p}(s))$ is a simple eigenvalue of $B(s)$ analytic in $s$ and that the components of $\mathbf{q}(s)$, the unique right eigenvector in $\Delta^{0}$ of $B(s)$ corresponding to $\rho(s)$, are also analytic functions of $s$. We display these quantities by the relation

$$
B(\mathbf{p}(s)) \mathbf{q}(s)=\rho(s) \mathbf{q}(s) \quad \text { where } \rho(s)=\rho(B(s)) .
$$

We hereafter use the notation

$$
\begin{aligned}
\dot{\mathbf{p}}(s) & =\frac{d \mathbf{p}(s)}{d s}=\left(\frac{d p_{1}(s)}{d s}, \ldots, \frac{d p_{r}(s)}{d s}\right), \\
\dot{\mathbf{q}}(s) & =\left(\frac{d q_{1}(s)}{d s}, \ldots, \frac{d q_{r}(s)}{d s}\right), \\
\ddot{\mathbf{p}}(s) & =\frac{d^{2} \mathbf{p}}{d s^{2}}(s) \quad \text { and } \quad \ddot{\mathbf{q}}(s)=\frac{d^{2} \mathbf{q}}{d s^{2}}(s),
\end{aligned}
$$

which we abbreviate to $\dot{\mathbf{p}}, \dot{\mathbf{q}}, \ddot{\mathbf{p}}$ and $\ddot{\mathbf{q}}$ at $s=0$.

Since $\langle\mathbf{p}(s), \mathbf{u}\rangle=\langle\mathbf{q}(s), \mathbf{u}\rangle=1$, we have

$$
\langle\dot{\mathbf{p}}(s), \mathbf{u}\rangle=\langle\ddot{\mathbf{p}}(s), \mathbf{u}\rangle=\langle\dot{\mathbf{q}}(s), \mathbf{u}\rangle=\langle\ddot{\mathbf{q}}(s), \mathbf{u}\rangle \equiv 0 .
$$


Observe that the transpose matrix $B(\mathbf{p})^{\prime}$ of $B(\mathbf{p})$ applied to $\xi$ is

$$
\xi B(\mathbf{p})=B(\mathbf{p})^{\prime} \xi=M \mathbf{p} \circ \xi+M(\mathbf{p} \circ \xi) .
$$

Choosing $\boldsymbol{\xi}=\mathbf{z}=\mathbf{q} / \mathbf{p}$ with $\mathbf{p}=\mathbf{p}(s)$ and $\mathbf{q}=\mathbf{q}(s)$ verifying (C.1), we get

$$
\mathbf{z} B(\mathbf{p})=\rho(\mathbf{p}) \mathbf{z} \text {. }
$$

Indeed,

$$
\mathbf{z} B(\mathbf{p})=(M \mathbf{p}) \circ \frac{\mathbf{q}}{\mathbf{p}}+M \mathbf{q}=\frac{1}{\mathbf{p}} \circ(M \mathbf{p} \circ \mathbf{q}+\mathbf{p} \circ M \mathbf{q})=\frac{1}{\mathbf{p}} \circ B(\mathbf{p}) \mathbf{q}=\rho(\mathbf{p}) \mathbf{z} .
$$

Furthermore, we have

$$
(1 / \mathbf{z}) B(\mathbf{q})=\rho(\mathbf{p}) \mathbf{u}
$$

In fact,

$$
\begin{aligned}
\frac{1}{\mathbf{z}} B(\mathbf{q}) & =B(\mathbf{q})^{\prime} \frac{1}{\mathbf{z}}=M \mathbf{q} \circ \frac{\mathbf{p}}{\mathbf{q}}+M\left(\mathbf{q} \circ \frac{\mathbf{p}}{\mathbf{q}}\right) \\
& =\frac{1}{\mathbf{q}} \circ[\mathbf{p} \circ M \mathbf{q}+M \mathbf{p} \circ \mathbf{q}]=\frac{1}{\mathbf{q}} \circ B(\mathbf{p}) \mathbf{q}=\rho(\mathbf{p}) \mathbf{u} .
\end{aligned}
$$

Differentiating the identity (C.1) in $s$ produces

$$
\dot{B}(\mathbf{p}(s)) \mathbf{q}(s)+B(\mathbf{p}(s)) \dot{\mathbf{q}}(s)=\dot{\rho}(s) \mathbf{q}(s)+\rho(s) \dot{\mathbf{q}}(s),
$$

which reduces to

$$
B(\mathbf{q}(s)) \dot{\mathbf{p}}(s)+B(\mathbf{p}(s)) \dot{\mathbf{q}}(s)=\dot{\rho}(s) \mathbf{q}(s)+\rho(s) \dot{\mathbf{q}}(s) .
$$

On account of (C.4), we obtain, with $\mathrm{z}(s)=\mathbf{q}(s) / \mathbf{p}(s)$,

$$
\begin{aligned}
& \langle\mathbf{z}(s) B(\mathbf{q}(s)), \dot{\mathbf{p}}(s)\rangle+\rho(s)\langle\mathbf{z}(s), \dot{\mathbf{q}}(s)\rangle \\
& =\dot{\boldsymbol{\rho}}(s)\langle\mathbf{z}(s), \mathbf{q}(s)\rangle+\rho(s)\langle\mathbf{z}(s), \dot{\mathbf{q}}(s)\rangle
\end{aligned}
$$

or

$$
\langle\mathbf{z}(s) B(\mathbf{q}(s)), \dot{\mathbf{p}}(s)\rangle=\dot{\rho}(s)\langle\mathbf{z}(s), \mathbf{q}(s)\rangle .
$$

Note also, on the basis of (C.5), that

$$
\langle(1 / \mathbf{z}(s)) B(\mathbf{q}(s)), \dot{\mathbf{p}}(s)\rangle=\rho(s)\langle\mathbf{u}, \dot{\mathbf{p}}(s)\rangle=0,
$$

the last equation resulting from (C.3).

We are now prepared to prove Theorem 6.1.

\section{Characterization of an equilibrium.}

Necessity. Let $\mathbf{p}^{*}=\mathbf{p}(0)$ be an equilibrium of $(6.4)$ in $\Delta^{0}$ so that $M \mathbf{p}^{*}=w^{*} \mathbf{u}$. Then because $M$ is positive, equation (C.1) for $s=0$, namely $B\left(\mathbf{p}^{*}\right) \mathbf{q}^{*}=\lambda^{*} \mathbf{q}^{*}$ where $\mathbf{q}^{*}=\mathbf{q}(0)$ and $\lambda^{*}=\rho(0)$, requires $\mathbf{q}^{*}=\mathbf{p}^{*}$ and $\lambda^{*}=2 w^{*}=$ $2\left\langle M \mathbf{p}^{*}, \mathbf{p}^{*}\right\rangle$. Thus, we have $\mathbf{z}^{*}=\mathbf{q}^{*} / \mathbf{p}^{*}=\mathbf{u}$, implying $\mathbf{u} B\left(\mathbf{p}^{*}\right)=2 w^{*} \mathbf{u}$, and (C.7) for $s=0$ indicates that $\dot{\rho}(0)=0$.

Sufficiency. If $\mathbf{p}^{*} \in \Delta^{0}$ furnishes a critical point for $\rho(\mathbf{p})$, i.e., $\dot{\rho}\left(\mathbf{p}^{*}\right)=0$, equations (C.7) and (C.8) for $s=0$ reduce to

$$
\left\langle\mathbf{z}^{*} B\left(\mathbf{q}^{*}\right), \boldsymbol{\eta}\right\rangle=0 \text { and }\left\langle\left(1 / \mathbf{z}^{*}\right) B\left(\mathbf{q}^{*}\right), \boldsymbol{\eta}\right\rangle=0,
$$


A direct analysis reveals that a sufficient condition for the polymorphism $\left\{\mathbf{p}^{*}, \mathbf{p}^{*}\right\}$ to be locally stable is that it satisfy the inequality

$$
\left|1+\left(\frac{1}{2}+\frac{b w_{F}\left(\mathbf{p}^{*}\right)}{2\left(a+b w_{F}\left(\mathbf{p}^{*}\right)\right)}\right) \lambda\right|<1
$$

for all eigenvalues $\lambda$ different from unity of the matrix transformation

$$
F^{*} \xi=\frac{\mathbf{p}^{*} \circ \mathbf{F}}{w_{F}\left(\mathbf{p}^{*}\right)} \xi .
$$

If (6.29) is strictly violated for one of the $\lambda$, then $\left\{\mathbf{p}^{*}, \mathbf{p}^{*}\right\}$ is not stable.

Appendix A. For positive vectors we use the notation $\mathbf{x} / \mathbf{y}=$ $\left(x_{1} / y_{1}, x_{2} / y_{2}, \ldots, x_{r} / y_{r}\right)$ and $\sqrt{x}=\left(\sqrt{x_{1}}, \sqrt{x_{2}}, \ldots, \sqrt{x_{r}}\right)$. Let $\mathbf{p}$ be a positive frequency state $\left(\mathbf{p} \in \Delta^{0}\right)$ and consider the functional

$$
G(\mathbf{x} ; \mathbf{p})=\prod_{i=1}^{r}\left(\frac{x_{i}}{w_{i}(\mathbf{x})}\right)^{p_{i}}, \quad w_{i}(\mathbf{x})=\sum_{j=1}^{r} m_{i j} x_{j}
$$

for $\mathbf{x}>0$ and $M=\left\|m_{i j}\right\|$ a symmetric positive matrix. We define

$$
\varphi(\mathbf{p})=\max _{\mathbf{x}>0} \sum_{i=1}^{r} p_{i} \log \frac{x_{i}}{w_{i}(\mathbf{x})}=\max _{\mathbf{x}>0} \log G(\mathbf{x}, \mathbf{p}) .
$$

The Donsker-Varadhan theory (1975), or, equivalently, the results of Friedland and Karlin (1975), affirms that for each $p$ the maximum of (A.1) is unique (up to a scale factor) achieved for $\mathbf{x}(\mathbf{p}) \in \Delta^{0}$ provided $D_{(M \mathbf{x})}^{-1} M D_{\mathbf{x}}$ has $\mathbf{p}$ as a left eigenvector of eigenvalue 1 . This relationship is a homeomorphic (actually analytic) mapping $\mathbf{p} \rightarrow \mathbf{x}(\mathbf{p})$ of $\Delta^{0}$ onto $\Delta^{0}$.

Observe that $D_{(M \mathbf{x})}^{-1} M D_{\mathbf{x}}$ has $\mathbf{u}=(1, \ldots, 1)$ as a right eigenvector for eigenvalue 1 . We may inquire if there is a fixed point

$$
\mathbf{p} \rightarrow \mathbf{x}(\mathbf{p})=\mathbf{p} \text {. }
$$

LEMMA A.1. $\mathbf{p}^{*}$ is a fixed point of the mapping (A.2) if and only if $\mathbf{p}^{*}$ is a polymorphic equilibrium of (6.4), i.e., $M \mathbf{p}^{*}=w^{*} \mathbf{u}$ with $w^{*}=\left\langle M \mathbf{p}^{*}, \mathbf{p}^{*}\right\rangle$.

Proof. Suppose p* $=\mathrm{p}^{*}\left(1 / M \mathrm{p}^{*}\right) M \circ \mathrm{p}^{*}$ or, equivalently, $M\left(\mathrm{p}^{*} / M \mathrm{p}^{*}\right)=\mathbf{u}$.

Consider now the vector $\mathrm{w}^{*}$ having coordinates $w_{j}^{*}=\left(M \mathrm{p}^{*}\right)_{j}$. By concavity of the square root function we have

$$
\sum_{j=1}^{r} m_{i j} \frac{p_{j}^{*}}{w_{j}^{*}} \sqrt{w_{j}^{*}} \leqslant \sqrt{\sum_{j=1}^{r} m_{i j} \frac{p_{j}^{*}}{w_{j}^{*}} w_{j}^{*}}=\sqrt{w_{i}^{*}}
$$

with strict inequality unless $w_{j}^{*} \equiv$ constant. If $\mathbf{w}^{*}$ is not a constant vector, we deduce from (A.3) that $\rho\left(M \circ \mathbf{p}^{*} / M \mathbf{p}^{*}\right)<1$. However, $\mathbf{p}^{*}\left(M \circ \mathbf{p}^{*} / M \mathbf{p}^{*}\right)=\mathbf{p}^{*}$, so $\rho\left(D_{M \mathbf{p}^{*}}^{-1} M D_{\mathbf{p}^{*}}\right)=1$. This contradiction can be averted only if $\left\{w_{j}^{*}\right\}_{1}^{r}$ is a constant vector or $M \mathbf{p}^{*}=w^{*} \mathrm{u}$, indicating that $\mathrm{p}^{*}$ is a polymorphism, as was to be shown.

The analysis of the concave function

$$
\psi(\mathbf{p})=\min _{\mathbf{x}>0}\left(\sum_{i} p_{i} \frac{(M \mathbf{x})_{i}}{x_{i}}\right)
$$


parallel to (A.1) shows that for $\mathbf{p} \in \Delta^{0}$ the minimum is uniquely attained at $\mathbf{x}$ that satisfies $\mathbf{p} D_{\mathbf{x}}^{-1} M D_{\mathbf{x}}^{2} D_{M \mathbf{x}}^{-1}=\mathbf{p}$. In the case that $M$ is symmetric we directly verify that $\mathbf{x}=\sqrt{\mathbf{p}}$, which implies

$$
\psi(\mathbf{p})=\sum_{i, j} m_{i j} \sqrt{p_{i}} \sqrt{p_{j}}
$$

is a concave function of $\mathbf{p}$. Moreover, for $M$ symmetric with one positive and $r-1$ negative eigenvalues we deduce in the presence of $(6.18 \mathrm{a})$ that $Q(\mathbf{p})=$ $\sum_{i, j=1}^{r} m_{i j} p_{i} p_{j}$ is also concave on $\Delta$.

When $\mathbf{p}^{*}$ is a stable polymorphism we find that $\Pi_{i=1}^{r}\left(w_{i}(\mathbf{p}) / w_{i}^{*}\right)^{p_{i}^{*}}$ exhibits a local strict maximum at $\mathbf{p}=\mathbf{p}^{*}$ relative to $\Delta$. Indeed,

$$
\prod_{i=1}^{r} w_{i}^{p_{i}^{*}} \leqslant \sum_{i=1}^{r} p_{i}^{*} w_{i}=\left\langle\mathbf{p}^{*}, M \mathbf{p}\right\rangle=\left\langle\mathbf{p}^{*} M, \mathbf{p}\right\rangle=w^{*}=\prod_{i=1}^{r}\left(w_{i}^{*}\right)^{p_{i}^{*}}
$$

Consider next the feasibility of the inequality

$$
\prod_{i=1}^{r} w_{i}^{p^{*}} \geqslant w=\sum_{i=1}^{r} p_{i} w_{i}>\prod_{i=1}^{r} w_{i}^{p_{i}}
$$

when $\mathbf{p}^{*}$ is a stable equilibrium of (6.4).

Passing to logarithms this inequality reduces to

$$
\sum_{i=1}^{r} p_{i}^{*} \log w_{i}-\log \left(\sum_{i=1}^{r} p_{i} w_{i}\right)>0 .
$$

Writing $\mathbf{p}=\mathbf{p}^{*}+\boldsymbol{\xi}$, stipulating $\langle\boldsymbol{\xi}, \mathbf{u}\rangle$ and $\|\boldsymbol{\xi}\|$ small, we expand both logarithms, noting $w_{i}(\mathbf{p})=w^{*}+\eta_{i}, \eta_{i}=w_{i}(\xi)$ and $\left\langle\mathbf{p}^{*}, \eta\right\rangle=\left\langle\mathbf{p}^{*}, M \xi\right\rangle=$ $w^{*}\langle\mathbf{u}, \xi\rangle=0$, to obtain

$$
\begin{aligned}
& \sum_{i=1}^{r} p_{i}^{*} \log w_{i}-\log \left(\sum_{i=1}^{r} p_{i} w_{i}\right) \\
& =-\frac{1}{2\left(w^{*}\right)^{2}} \sum_{i=1}^{r} p_{i}^{*}\left(w_{i}(\xi)\right)^{2}-\frac{w(\xi)}{w^{*}}+O\left(|\xi|^{3}\right) .
\end{aligned}
$$

Now set $C=\sqrt{\mathbf{p}^{*}} \circ M \circ \sqrt{\mathbf{p}^{*}}$. This symmetric matrix has its maximal eigenvalue equal to $w^{*}$ with corresponding eigenvector $\sqrt{\mathbf{p}^{*}}$. We denote the other eigenvalues by $\mu_{j}$, and corresponding orthonormal eigenvectors by $\varphi_{j}, j=$ $2, \ldots, r$. Using the expansion, with the cognizance of $\langle\boldsymbol{\xi}, \mathbf{u}\rangle=0$,

$$
\frac{\xi}{\sqrt{\mathbf{p}^{*}}}=\left\langle\frac{\boldsymbol{\xi}}{\sqrt{\mathbf{p}^{*}}}, \sqrt{\mathbf{p}^{*}}\right\rangle \sqrt{\mathbf{p}^{*}}+\sum_{j=2}^{n}\left\langle\frac{\xi}{\sqrt{\mathbf{p}^{*}}}, \boldsymbol{\varphi}_{j}\right\rangle \boldsymbol{\varphi}_{j}=\sum_{j=2}^{n}\left\langle\frac{\boldsymbol{\xi}}{\sqrt{\mathbf{p}^{*}}}, \boldsymbol{\varphi}_{j}\right\rangle \boldsymbol{\varphi}_{j},
$$

leads to the identities

$$
\sum_{i=1}^{r} p_{i}^{*}\left(w_{i}(\xi)\right)^{2}=\left\langle C \frac{\xi}{\sqrt{\mathbf{p}^{*}}}, C \frac{\xi}{\sqrt{\mathbf{p}^{*}}}\right\rangle=\sum_{j=2}^{r} \mu_{j}^{2}\left[\left\langle\frac{\xi}{\sqrt{\mathbf{p}^{*}}}, \varphi_{j}\right\rangle\right]^{2}
$$

and

$$
w(\xi)=\left\langle C \frac{\xi}{\sqrt{\mathbf{p}^{*}}}, \frac{\boldsymbol{\xi}}{\sqrt{\mathbf{p}^{*}}}\right\rangle=\sum_{j=2}^{r} \mu_{j}\left\langle\frac{\boldsymbol{\xi}}{\sqrt{\mathbf{p}^{*}}}, \varphi_{j}\right\rangle^{2} .
$$


Combining into (A.6) yields

$$
-\frac{1}{2 w^{* 2}} \sum_{i=1}^{r} p_{i}^{*} w_{i}(\xi)^{2}-\frac{w(\xi)}{w^{*}}=\frac{1}{w^{*}} \sum_{j=2}^{r}\left[\left(-\mu_{j}\right)-\frac{\mu_{j}^{2}}{2 w^{*}}\right]\left\langle\frac{\xi}{\sqrt{\mathbf{p}^{*}}}, \varphi_{j}\right\rangle^{2} .
$$

Since $\left|\mu_{j}\right|<w^{*}$ (by the Frobenius Theory of positive matrices) and all $\mu_{j}<0$, $j=2, \ldots, r$ (by virtue of (6.18a)), the above quantity satisfies the inequality

$>c\|\boldsymbol{\xi}\|^{2}$ for some $c>0$ valid for all $\boldsymbol{\xi}$ in the linear manifold $\langle\boldsymbol{\xi}, \mathbf{u}\rangle=0$.

Examination of the proof reveals more. For a stable polymorphism the quadratic form

$$
-w(\xi)-\frac{1}{w^{*}} \sum_{i=1}^{r} p_{i}^{*}\left(w_{i}(\xi)\right)^{2}
$$

is strictly positive definite over the linear manifold $\mathscr{L}_{\mathbf{u}}=\{\boldsymbol{\xi},\langle\boldsymbol{\xi}, \mathbf{u}\rangle=0\}$.

The foregoing analysis shows that in the vicinity of a stable polymorphism $\mathbf{p}^{*}, H(\mathbf{p})=\prod_{i=1}^{r}\left(p_{i}\right)^{p_{i}^{*}}$ constitutes a local Lyapounov function for the transformation (6.4). In terms of the coordinates $p_{i}^{\prime}=p_{i} w_{i}(\mathbf{p}) / w(\mathbf{p})$, the inequality $H\left(\mathbf{p}^{\prime}\right)>H(\mathbf{p})$ reduces to

$$
\prod_{i=1}^{r}\left[w_{i}(\mathbf{p})\right]^{p_{i}^{*}}>w(\mathbf{p})=\prod_{i=1}^{r}[w(\mathbf{p})]^{p_{i}^{*}},
$$

valid for $\mathbf{p}$ near $\mathbf{p}^{*}, \mathbf{p} \neq \mathbf{p}^{*}$. The same result applies in the neighborhood of a stable boundary equilibrium. It is conjectured that $H(\mathbf{p})$ is a global Lyapounov function. The result is correct for $r=2$. have

An interesting consequence of (A.6) is that for a stable polymorphism $\mathbf{p}^{*}$ we

$$
\prod_{i=1}^{r} m_{k i}^{p_{i}^{*}}>m_{k k}, \quad k=1,2, \ldots, r
$$

Appendix B. Some consequences of the internal stability conditions (6.18). Let $M=\left\|m_{i j}\right\|_{1}^{r}$ be a viability matrix obeying

$$
\langle M \xi, \xi\rangle<0 \text { for all } \boldsymbol{\xi} \neq 0 \text { satisfying }\langle\boldsymbol{\xi}, \mathbf{u}\rangle=0,
$$

Define

$$
\tilde{m}_{i j}=m_{i j}-m_{i r}-m_{r j}+m_{r r}, \quad 1 \leqslant i, j \leqslant r-1,
$$

and enlarge $\tilde{M}$ to an $r \times r$ matrix $\tilde{\tilde{M}}$ by the assignment $\tilde{m}_{r j}=\tilde{m}_{i r}=0$ for all $i$, $j$. Let $\eta=\left(\eta_{1}, \eta_{2}, \ldots, \eta_{r-1}\right)$ be an arbitrary nonnull $(r-1)$-vector and extend $\eta$ to $\xi,\left(\eta_{1}, \eta_{2}, \ldots, \eta_{r-1}, \eta_{r}\right)$ by setting $\eta_{r}=-\sum_{i=1}^{r-1} \eta_{i}$ so that $\langle\xi, u\rangle=0$. It is easy to verify $\langle M \xi, \xi\rangle=\langle\tilde{\tilde{M}} \xi, \xi\rangle$, which in turn is equal to $\langle\tilde{M} \eta, \eta\rangle$.

These identifications in conjunction with (B.1) imply $\tilde{M}$ is negative definite. Therefore the diagonal elements of $\tilde{M}$ are negative. Since the foregoing analysis is invariant under relabeling of the indices, we may conclude that

$$
m_{i i}+m_{j j}<2 m_{i j} \text { for all } i \neq j \text {. }
$$


Summing (B.3) over all $i \neq j$, we obtain

$$
\bar{W}_{\text {het. }}=\frac{1}{r(r-1) / 2} \sum_{1 \leqslant j \leqslant r} m_{i j}>\bar{W}_{\text {hom. }}=\frac{1}{r} \sum_{k=1}^{r} m_{k k},
$$

that the average heterozygote fitness exceeds the average homozygote fitnesses (Ginsburg (1979)).

Using the fact that the mean fitness function $w(\mathbf{p})=\sum m_{i j} p_{i} p_{j}$ is strictly concave over the frequency simplex attaining its maximum at the stable polymorphic point, the weighted average comparison

$$
\frac{\sum_{i \neq j} m_{i j} p_{i}^{*} p_{j}^{*}}{\sum_{i \neq j} p_{i}^{*} p_{j}^{*}}>\frac{\sum_{k=1}^{r} m_{k k}\left(p_{k}^{*}\right)^{2}}{\sum_{k=1}^{r}\left(p_{k}^{*}\right)^{2}}
$$

for a stable polymorphic frequency state $\mathbf{p}^{*}=\left(p_{1}^{*}, p_{2}^{*}, \ldots, p_{r}^{*}\right)$ obtains.

Another variant of (B.5) is as follows. Let $\varphi=\left(\varphi_{1}, \varphi_{2}, \ldots, \varphi_{r}\right)$ be the unique positive eigenvector $M \varphi=\lambda^{*} \varphi$ (normalized so that $\sum_{i=1}^{r} \varphi_{i}^{2}=1$ ) corresponding to the maximal eigenvalue $\lambda^{*}$ of $M$. If $M$ satisfies (B.1) we also have

$$
m_{i i}-\frac{2 \sum_{k=1}^{r} m_{i k} \varphi_{k}}{\sum_{k=1}^{r} \varphi_{k}}+\frac{\sum_{i, j=1}^{r} m_{i j} \varphi_{i} \varphi_{j}}{\left(\sum_{k=1}^{r} \varphi_{k}\right)^{2}}<0, \quad i=1,2, \ldots, r .
$$

Averaging with respect to $\varphi_{i}$, we obtain

$$
\left(\sum_{i=1}^{r} \varphi_{i}\right)\left(\sum_{i=1}^{r} m_{i i} \varphi_{i}\right)<\sum_{i, j=1}^{r} m_{i j} \varphi_{i} \varphi_{j}
$$

The existence of a stable polymorphism $\mathbf{p}^{*}=\left(p_{1}^{*}, \ldots, p_{n}^{*}\right)$ implies that heterozygote fitness values cannot differ too much. In fact, Lewontin et al. (1978) established the following triangle inequality. For each heterozygote $\mathbf{A}_{i} \mathbf{A}_{j}(i \neq j)$ there exists some index $k$ (not necessarily all) with the property that $m_{i j}<m_{i k}+m_{k j}$. From this fact it follows that the maximal fitness coefficient cannot exceed twice the next largest fitness value. In general, this inequality cannot be improved. However, when

$$
\min _{k \neq l}\left(m_{k l}\right)>\max _{i}\left(m_{i i}\right)
$$

it is easy to prove:

If $M$ satisfies (B.8) and a stable polymorphism exists, then for each $i \neq j$ and every $k$,

$$
m_{i j}+\min \left(m_{i i}, m_{j j}\right)<m_{i k}+m_{k j}
$$

Appendix C. Proof of Theorem 6.1. We start with some preliminaries. For a polymorphic equilibrium $\mathrm{p}^{*}$, since $M \mathrm{p}^{*}=w^{*} \mathrm{u}, w^{*}=w\left(\mathrm{p}^{*}\right)$, we have $S^{\prime}\left(\mathrm{p}^{*}\right)=$ $I+M^{*}$ where $M^{*}=\left(\mathrm{p}^{*} \circ M\right) / w^{*}$. Observe that $M^{*} \mathbf{p}^{*}=\mathrm{p}^{*}$ and $\mathbf{u} M^{*}=\mathbf{u}$, and since $M^{*}$ is strictly positive, the spectral radius of $M^{*}$ is 1 . It is convenient to define

$$
M(\mathbf{p})=(\mathbf{p} / M \mathbf{p}) \circ M
$$


valid for every $\eta \in \mathscr{L}$, i.e., for all $\eta$ satisfying $\langle\eta, u\rangle=0$. Since $\mathscr{L}$ is $(n-1)$ dimensional, equations (C.9) are compatible only if $\mathbf{z}^{*} B\left(\mathbf{q}^{*}\right)=\alpha\left(1 / \mathbf{z}^{*}\right) B\left(\mathbf{q}^{*}\right)$ for some positive constant $\alpha$. Recall that $B\left(\mathbf{q}^{*}\right)$ is invertible, implying that $\mathbf{z}^{*}=\alpha / \mathbf{z}^{*}$; but this is feasible only if $\mathbf{q}^{*}=\sqrt{\alpha} \mathbf{p}^{*}$, actually $\mathbf{q}^{*}=\mathbf{p}^{*}$ since both are frequency vectors, and then Lemma C.1 yields the conclusion.

II. Stability criterion of an internal equilibrium. Let $\mathbf{p}^{*}=\mathbf{q}^{*}=\mathbf{p}(0)=\mathbf{q}(0)$ be a critical point of $\rho(\mathbf{p})$. Another differentiation of (C.6) evaluated at $s=0$ produces, under the notation of (C.2),

$$
B\left(\mathbf{p}^{*}\right) \ddot{\mathbf{p}}+B\left(\mathbf{p}^{*}\right) \ddot{\mathbf{q}}+2 \dot{\mathbf{p}} \circ M \grave{\mathbf{q}}+2 \dot{\mathbf{q}} \circ M \dot{\mathbf{p}}=\rho(0) \ddot{\mathbf{q}}+\ddot{\rho}(0) \mathbf{p}^{*}
$$

( $\ddot{\rho}(0)$ is the second derivative of $\rho(s)$ in the direction of $\eta$ evaluated at $s=0$ ). In this case, since $M \mathrm{p}^{*}=w^{*} \mathrm{u}$, we have $B\left(\mathrm{p}^{*}\right)=w^{*}\left(I+M^{*}\right)$, where we have introduced the notation

$$
M^{*}=\left(\mathrm{p}^{*} \circ M\right) / w^{*} .
$$

Reference to $\mathbf{u} B\left(\mathbf{p}^{*}\right)=2 w^{*} \mathbf{u}$, after taking the inner product of (C.10) with $\mathbf{u}$ in view of equations (C.3), gives

$$
\ddot{\rho}(0)=4\langle\dot{\mathbf{p}}, M \dot{\mathbf{q}}\rangle,
$$

and expressed in terms of the generalized inner product $\langle,\rangle_{\mathbf{d}}$ with $\mathbf{d}=\mathbf{p}^{*} / w^{*}$ we have

$$
\ddot{\rho}(0)=4\left\langle\dot{\mathbf{p}}, M^{*} \dot{\mathbf{q}}\right\rangle_{\mathbf{d}} .
$$

We next rewrite (C.6) at $s=0$ in the form (using (C.11))

$$
\left(I-M^{*}\right) \dot{\mathbf{q}}=\left(I+M^{*}\right) \dot{\mathbf{p}} \text {. }
$$

Both $I+M^{*}$ and $I-M^{*}$ are invertible on $\mathscr{L}=\{\xi ;\langle\xi, \mathbf{u}\rangle=0\}$ and leave this linear manifold invariant. Moreover, $M^{*}$ is symmetric in the inner product $\langle,\rangle_{d}$. Thus, every vector $\mathbf{x}$ in $\mathscr{L}$ can be represented, using the orthonormal basis $\left\{\varphi_{i}\right\}$ of eigenvectors of $M^{*}$ in $\mathscr{L}$, as

$$
\mathbf{x}=\sum_{i=1}^{r-1}\left\langle\mathbf{x}, \varphi_{i}\right\rangle_{\mathbf{d}} \varphi_{i}
$$

In particular,

$$
\dot{\mathbf{q}}=\sum_{i=1}^{r-1}\left\langle\dot{\mathbf{q}}, \varphi_{i}\right\rangle_{\mathrm{d}} \varphi_{i}, \quad \dot{\mathbf{p}}=\sum_{i=1}^{r-1}\left\langle\dot{\mathbf{p}}, \varphi_{i}\right\rangle_{\mathrm{d}} \varphi_{i}
$$

Equation (C.14) with $M^{*} \varphi_{i}=\lambda_{i} \varphi_{i}$ implies

$$
\left(1-\lambda_{i}\right)\left\langle\dot{\mathbf{q}}, \varphi_{i}\right\rangle_{\mathbf{d}}=\left(1+\lambda_{i}\right)\left\langle\dot{\mathbf{p}}, \varphi_{i}\right\rangle_{\mathbf{d}},
$$

where $\lambda_{i}$ are all real and $\left|\lambda_{i}\right|<1$. Expanding (C.13) via (C.15) and using (C.16) we get

$$
\ddot{\rho}(0)=4 \sum_{i=1}^{r-1} \lambda_{i}\left[\left\langle\dot{\mathbf{p}}, \varphi_{i}\right\rangle_{\mathrm{d}}\right]^{2} \frac{1+\lambda_{i}}{1-\lambda_{i}}
$$


As $\dot{\mathbf{p}}=\eta$ is an arbitrary vector in $\mathscr{L}$, we may conclude that $\ddot{\rho}(0)<0$ for all directions if and only if $\lambda_{i}<0, i=1,2, \ldots, r-1$. Thus, $\mathbf{p}^{*}$ is locally stable if and only if $\rho(\mathbf{p})$ exhibits a (strict) local maximum at $\mathbf{p}^{*}$.

This completes the proof of Theorem 6.1.

7. Mathematical models of sex ratio evolution. The results of this section were developed in collaboration with Sabin Lessard; see Karlin and Lessard $(1983,1984)$ for elaborations, proofs and extensions.

Sex determination governed at a single autosomal locus with probabilities of being male or female depending on the zygote genotype was first given a theoretical formulation in Shaw (1958). A two-allele model was studied by Eshel (1975). A variant of this model, dealt with in Nur (1974) and Uyenoyama and Bengtsson (1979), admitting maternal (or paternal) genotype controls on the expected brood sex ratio, led to similar qualitative conclusions. Eshel and Feldman (1982) considered generalizations to multiallelic cases (see also references therein).

In this section we will elaborate, for panmictic populations, a series of sex-determining genetic models controlled at an autosomal locus entailing multiallelic variants allowing for sex expression to depend on the zygote genotype, or one or both of the parental genotypes.

MODEL I. An autosomal multiallele sex-determination system based on the genotype of the offspring. Consider a bisexual infinite population with $r$ possible alleles $A_{1}, A_{2}, \ldots, A_{r}$ at an autosomal locus primarily responsible for sex determination. We denote the frequency of genotype $A_{i} A_{j}$ in the female population by $2 p_{i j}$ when $i \neq j$, and by $p_{i i}$ when $i=j$. The frequency of allele $\mathrm{A}_{i}$ is $p_{i}=\sum_{j=1}^{r} p_{i j}$. The quantities $2 q_{i j}, q_{i i}$ and $q_{i}$ are defined analogously with respect to the male population. We assume discrete generations, random mating, Mendelian segregation and equal fertility for all mating types. Let $m_{i j}$ be the probability for an $A_{i} A_{j}$ individual to be a male and, concomitantly, $1-m_{i j}$ that of being a female. Clearly, $0 \leqslant m_{i j}=m_{j i} \leqslant 1$. We refer to $M=\left\|m_{i j}\right\|_{i, j=1}^{r}$ as the sex-determination coefficient matrix.

The case of $0<m_{i j}<1$ may reflect the effects of modifier genes coupled to prenatal and/or neonatal interactions. Where $m_{i j}=1$ or 0 , the sex phenotype is exactly determined such that the collection of all genotypes $\mathrm{A}_{i} \mathrm{~A}_{j}$ partition into two groups $\mathscr{G}_{m}$ and $\mathscr{G}_{f}$ where every individual of types $\mathscr{G}_{m}$ and $\mathscr{G}_{f}$ is unambiguously male and female, respectively. We refer to this situation as dichotomous (exact) genotypic sex determination. Corresponding multitype decompositions are appropriate for studies of incompatibility systems in plants and in elucidating consequences of nonrandom mating patterns.

Returning to the general model, the genotype frequencies over two successive generations obey the recursion relations

$$
q_{i j}^{\prime}=\frac{m_{i j}\left(p_{i} q_{j}+p_{j} q_{i}\right)}{2 w}, \quad p_{i j}^{\prime}=\frac{\left(1-m_{i j}\right)\left(p_{i} q_{j}+p_{j} q_{i}\right)}{2(1-w)}
$$

where $w=\sum_{i, j=1}^{r} m_{i j} p_{i} q_{j}$ is the proportion of males, the sex ratio in the total population for the given allelic frequency state. 
System (7.1) can be converted into the following recurrence equations for the frequency vectors $\mathbf{p}=\left(p_{1}, p_{2}, \ldots, p_{r}\right)$ and $\mathbf{q}=\left(q_{1}, q_{2}, \ldots, q_{r}\right)$ and those of the subsequent generation $\mathbf{p}^{\prime}$ and $\mathbf{q}^{\prime}$.

$$
\begin{gathered}
\mathbf{q}^{\prime}=\frac{\mathbf{p} \circ M \mathbf{q}+\mathbf{q} \circ M \mathbf{p}}{2 w}, \\
\mathbf{p}^{\prime}=\frac{\mathbf{p} \circ(U-M) \mathbf{q}+\mathbf{q}^{\circ}(U-M) \mathbf{p}}{2(1-w)}=\frac{\mathbf{p}+\mathbf{q}-\mathbf{q}^{\prime} 2 w}{2(1-w)},
\end{gathered}
$$

where

$$
w=\langle\mathbf{p}, M \mathbf{q}\rangle=\sum_{i=1}^{r} p_{i}(M \mathbf{q})_{i}=\sum_{i, j=1}^{r} m_{i j} p_{i} q_{j}
$$

and $U$ denotes the matrix with all unit entries.

At equilibrium for (7.2) $\mathbf{q}^{\prime}=\mathbf{q}, \mathbf{p}^{\prime}=\mathbf{p}$, we immediately have from (7.2b)

$$
(1-2 w) \mathbf{q}=(1-2 w) \mathbf{p} .
$$

An equilibrium with $\mathbf{q}^{*}=\mathbf{p}^{*}$ is called a symmetric equilibrium exhibiting identical allelic frequencies in the male and female populations. (Symmetric equilibria are also called genotypic equilibria.) An even-sex ratio equilibrium (also called phenotypic equilibrium) has $\hat{w}=1 / 2$, that is, maintains an equal representation of males and females in the population.

The transformation equations (7.2) are recognized as a case of the dynamical system (6.3) describing the allele frequency changes for a two-sex viability model. Formally the sex-determination model where an offspring of genotype $\mathrm{A}_{i} \mathrm{~A}_{j}$ is male (female) with probability $m_{i j}\left(\left(1-m_{i j}\right)\right)$ is equivalent to a sex-differentiated viability model with viability matrices $M=\left\|m_{i j}\right\|$ and $F=$ $\left\|1-m_{i j}\right\|=U-M$ for males and females, respectively. The fact that $M$ and $F=U-M$ in the sex-determination model are generated from a single generic matrix $M$ allows a more tractable analysis of the system.

The following facts are fundamental to our later analysis. A symmetric equilibrium $\left\{\mathbf{p}^{*}, \mathbf{p}^{*}\right\}$ of (7.2) (abbreviated by $\left.\mathbf{p}^{*}\right)$ must verify $S \mathbf{p}^{*}=\mathbf{p}^{*}$, where

$$
S \mathbf{p}=\mathbf{p} \circ M \mathbf{p} /\langle\mathbf{p}, M \mathbf{p}\rangle,
$$

that is, $S$ is the transformation for the classical one-locus multiallele viability model with $M$ as a viability matrix (see (6.4)). An even-sex ratio equilibrium state $\{\tilde{\mathbf{q}}, \tilde{\mathbf{p}}\}$ is characterized by the relation

$$
\tilde{\mathbf{q}}=\tilde{\mathbf{p}} \circ M \tilde{\mathbf{q}}+\tilde{\mathbf{q}} \circ M \tilde{\mathbf{p}} .
$$

This relation entails a one-to-one sex ratio. The proof of this statement is as follows. Summing over the coordinates in (7.5) we get $1=\langle\mathbf{u}, \tilde{\mathbf{q}}\rangle=2\langle\tilde{\mathbf{p}}, M \tilde{\mathbf{q}}\rangle$, where $\mathbf{u}$ is the vector of all unit components and therefore $\tilde{w}=\langle\tilde{\mathbf{p}}, M \tilde{\mathbf{q}}\rangle=1 / 2$; the equations in (7.2) exhibiting $\mathbf{q}^{\prime}=\mathbf{q}=\tilde{\mathbf{q}}$ and $\mathbf{p}^{\prime}=\mathbf{p}=\tilde{\mathbf{p}}$ obtain on account of (7.5) and the fact that $\tilde{w}=1 / 2$.

Recall the matrix ((6.21))

$$
B(\mathbf{p})=D_{\mathbf{p}} M+D_{M \mathbf{p}}
$$

and the eigenvalue-eigenvector identity $((6.22))$

$$
B(\mathbf{p}) \mathbf{y}(\mathbf{p})=\rho(\mathbf{p}) \mathbf{y}(\mathbf{p}) .
$$


Comparing with (7.5), an even-sex ratio equilibrium represented by $\{\tilde{\mathbf{q}}, \tilde{\mathbf{p}}\}$, having both $\tilde{\mathbf{q}}$ and $\tilde{\mathbf{p}}$ interior to $\Delta$, exists if and only if $\rho(\tilde{\mathbf{p}})=1$, and then the corresponding $\mathbf{y}(\tilde{\mathbf{p}})$ is $\tilde{\mathbf{q}}$. These conditions can be extended by continuity allowing $\tilde{\mathbf{p}}$ and $\tilde{\mathbf{q}}$ on the boundary of $\Delta$.

We next describe the stability conditions for the symmetric and even-sex ratio equilibrium states.

Symmetric equilibria. Consider a symmetric equilibrium $\left\{\mathbf{p}^{*}, \mathbf{p}^{*}\right\}$ for the sex-determination model (7.2) represented by $\mathbf{p}^{*}$ for brevity. To avoid situations involving special relations among the parameters $\left\{m_{i j}\right\}$ of no special import, it will be appropriate, unless indicated otherwise, to impose the following genericy assumption.

Definition 7.1. A sex-determination matrix $M$ is said to be symmetrically generic if:

(i) $M$ and $U-M$ are each generic (in the sense of Definition 6.1);

(ii) $\left\langle\mathbf{p}^{*}, M \mathbf{p}^{*}\right\rangle \neq 1 / 2$ for every symmetric equilibrium $\left\{\mathbf{p}^{*}, \mathbf{p}^{*}\right\}$ of (7.2). In particular, $m_{i i} \neq 1 / 2$ for every $i$.

Requirement (ii) guarantees that a symmetric equilibrium state does not confer an even-sex ratio.

The next theorem indicates when a symmetric equilibrium is locally stable in the sex-determination model.

THEOREM 7.1. A symmetric interior equilibrium $\left\{\mathbf{p}^{*}, \mathbf{p}^{*}\right\}$ with $\mathbf{w}^{*}$ $=\left\langle\mathbf{p}^{*}, M \mathbf{p}^{*}\right\rangle<1 / 2$ is stable in the sex-determination model (7.2) if and only if $\mathbf{p}^{*}$ is stable for the one-locus viability matrix $M$ of the dynamical equations (6.4).

Analogously, if $w^{*}>1 / 2,\left\{\mathbf{p}^{*}, \mathbf{p}^{*}\right\}$ is a stable symmetric equilibrium for the sex-determination model (7.2) if and only if $\mathbf{p}^{*}$ is stable for the viability matrix $U-M$.

COROLlaRY 7.1. Where $\mathbf{p}^{*}$ is stable for the one-sex random mating selection system (6.4) with associated viability matrix $M$ and $w^{*}>1 / 2,\left\{\mathbf{p}^{*}, \mathbf{p}^{*}\right\}$ is unstable in the sex-determination model (7.2).

The proof of Theorem 7.1 is quite simple by appealing to the analysis set forth in (6.25)-(6.28). In the case at hand, the equilibrium point $\left\{\mathbf{p}^{*}, \mathbf{p}^{*}\right\}$ is stable if and only if the dominant eigenvalue of the gradient matrix of the system (6.3) at $\left\{\mathbf{p}^{*}, \mathbf{p}^{*}\right\}$ which is the $2 r \times 2 r$ matrix

$$
\left(\begin{array}{ll}
P & P \\
Q & Q
\end{array}\right)
$$

with

$$
\left.P=\frac{1}{2} I+\frac{1}{2} \frac{\mathrm{p} \circ M}{w^{*}}, \quad Q=\frac{1}{2} I+\frac{1}{2} \frac{\mathbf{p}^{*} \circ(U-M)}{\left(1-w^{*}\right)}\right)
$$

has all its eigenvalues relative to the domain $\mathscr{L} \otimes \mathscr{L}=\{\{\boldsymbol{\xi}, \eta\},\langle\boldsymbol{\xi}, \mathbf{u}\rangle=0$, $\langle\boldsymbol{\eta}, \mathbf{u}\rangle=0\}$ of magnitude less than one. The relevant eigenvalues (by Lemma 6.1) are those of

$$
P+Q=I+\frac{\left(1-2 w^{*}\right)}{2\left(1-w^{*}\right) w^{*}} D_{\mathbf{p}^{*}} M \text { on } \mathscr{L}_{\mathbf{u}}
$$


The above matrix resembles the gradient matrix at $\mathbf{p}^{*}$ for the one-locus selection model associated with viability matrix $M$ except for the constant multiplier $\left(1-2 w^{*}\right) / 2\left(1-w^{*}\right)$ (compare to (6.20)). It follows that if $\mathrm{p}^{*} \in \Delta^{0}$ is a stable equilibrium of (6.4) provided that $M^{*}=\left(p^{*} \circ M\right) / w^{*}$ has only negative eigenvalues of magnitude less than 1 with respect to $\mathscr{L}_{\mathrm{u}}$ and $w^{*}<1 / 2$, then all eigenvalues of $P+Q$ are also of magnitude less than 1 relative to $\mathscr{L} \otimes \mathscr{L}$, and conversely.

The stability analysis for a symmetric boundary equilibrium $\{\hat{\mathbf{p}}, \hat{\mathbf{p}}\}$ of system (7.2) is as follows.

THEOREM 7.2. A symmetric equilibrium $\{\hat{\mathbf{p}}, \hat{\mathbf{p}}\}$ with $\hat{w}<1 / 2$ is stable for the sex-determination model (7.2) if and only if:

(i) The eigenvalues of $(\hat{\mathbf{p}} \circ M) / \hat{w}$ in the face of $\hat{\mathbf{p}}$, i.e., with respect to $\mathscr{L}_{\vec{a}}=$ $\left\{\xi: \Sigma_{i \in l(\hat{\beta})} \xi_{i}=0\right\}$ are all negative, and

$$
\frac{(M \hat{\mathbf{p}})_{j}}{2 \hat{w}}+\frac{((U-M) \hat{\mathbf{p}})_{j}}{2(1-\hat{w})}<1, \quad j \notin l(\hat{\mathbf{p}}) ;
$$

or, equivalently, since $\hat{w}<1 / 2$,

$$
(M \hat{p})_{j}<\hat{w} \text { for } j \notin l(\mathbf{p}) .
$$

When $\hat{w}>1 / 2$, then the ascertainment of stability for $\{\hat{\mathbf{p}}, \hat{\mathbf{p}}\}$ in the sex-determination model (7.2) corresponds to the stability criteria for the one-locus viability matrix $U-M$ instead of $M$.

Thus, a symmetric equilibrium (boundary or interior) of sex ratio $\hat{w}<1 / 2$ $(\hat{w}>1 / 2)$ is stable for the sex-determination model of (7.2) if and only if it is stable for the one-locus random mating multiallele system with viability matrix $M(U-M)$, respectively.

Properties of even-sex ratio equilibria of the sex-determination model. We stipulate the genericy assumption of Definition 7.1 in force. Let $\{\tilde{\mathbf{q}}, \tilde{\mathbf{p}}\}$ be an equilibrium for the sex determination (7.2) with

$$
\langle\tilde{\mathbf{q}}, M \tilde{\mathbf{p}}\rangle=1 / 2 \text {. }
$$

We pointed out in connection with (7.5) and (7.6) that an interior even-sex ratio equilibrium is completely characterized by the eigenvalue-one equation

$$
B(\tilde{\mathbf{p}}) \tilde{\mathbf{q}}=\tilde{\mathbf{q}},
$$

where the matrix $B(\mathbf{p})=D_{M p}+D_{\mathbf{p}} \circ M$ is specified in (7.6). When (7.9) and (7.10) prevail, necessarily $\tilde{\mathbf{q}} \neq \tilde{\mathbf{p}}$, owing to the genericy postulate of Definition 7.1. A boundary even-sex ratio equilibrium $\{\tilde{\mathbf{q}}, \tilde{\mathbf{p}}\}$ segregating alleles of indices $l(\tilde{\mathbf{p}})$ also satisfies (7.10) with $\tilde{\mathbf{q}}$ interior to the same face as $\tilde{\mathbf{p}}$.

From the discussion of (7.2) and (7.7), we know that an interior even-sex ratio equilibrium exists if and only if $\rho(\tilde{\mathbf{p}})=\rho(B(\tilde{\mathbf{p}}))=1$ and $\tilde{\mathbf{q}}$ is the corresponding unique normalized positive right eigenvector for $B(\tilde{\mathbf{p}})$. The basic result reported next concerns the existence of even-sex ratio equilibrium states. Such equilibria comprise a continuum when present as indicated in the following theorem. 
THEOREM 7.3. Suppose $M$ is symmetrically generic, i.e., the conditions of Definition 7.1 hold.

(i) Even-sex ratio equilibria of the sex-determination model (7.2), when they exist, are part of an equilibrium surface (one or several) each of dimension $r-2$ in the simplex $\Delta$ of dimension $r-1$. Only in the case of two alleles are the even-sex ratio equilibria isolated points.

(ii) A stable symmetric equilibrium (interior or on the boundary) which is stable for the corresponding viability model (having the transformation equations (6.4)) cannot coexist with any even-sex ratio equilibrium segregating the same alleles.

(iii) There may coexist one or several surfaces of even-sex ratio equilibria.

(iv) Two symmetric equilibria $\hat{\mathbf{p}}_{\alpha}$ and $\hat{\mathbf{p}}_{\beta}$ with sex-ratio $\hat{w}_{\alpha}<1 / 2$ and $\hat{w}_{\beta}>1 / 2$, respectively, are separated by at least one even-sex ratio equilibrium surface, which by (ii) cannot intersect the interior of $l\left(\hat{\mathbf{p}}_{\alpha}\right)$ (the index set of $\hat{\mathbf{p}}_{\alpha}$ ) if $\hat{\mathbf{p}}_{\alpha}$ is stable and analogously for $\hat{\mathbf{p}}_{\beta}$.

COROLlARY 7.3. If there exists a stable symmetric polymorphic equilibrium $\mathbf{p}^{*}$ for the model (7.2), Then no even-sex ratio equilibrium exists and $\mathbf{p}^{*}$ is the unique stable equilibrium for the sex-determination model.

An analytic formula for the surface (or surfaces) of even-sex ratio equilibria is described as the set of all $p \in \Delta$ satisfying

$$
\varphi(\mathbf{p})=\operatorname{det}\left\|\left(\sum_{k=1}^{r} p_{k} m_{k j}\right) \delta_{j i}+p_{i} m_{i j}-\delta_{i j}\right\|_{i, j=1}^{r}=0
$$

$\left(\delta_{i j}=1\right.$ if $i=j ; 0$ when $\left.i \neq j\right)$. Note that the determinant is an algebraic function of degree $r$ in the variables $\left(p_{1}, p_{2}, \ldots, p_{r}\right)$.

Note that if there exists a stable symmetric polymorphic equilibrium $\mathbf{p}^{*}$ then no even-sex ratio equilibrium can exist and $\mathbf{p}^{*}$ is the unique stable equilibrium for the sex-determination model. It is worth emphasizing that stable symmetric equilibrium states and even-sex ratio equilibria can coexist, but then the symmetric and unsymmetric equilibrium states do not represent the identical set of alleles.

Stable equilibrium possibilities for dichotomous sex ratio determination. The following general result underscores the central role of the one-to-one sex ratio when the sex phenotype is controlled at a single multiallele locus with exact genotypic sex ascertainment.

THEOREM 7.4. Suppose all genotypes involving $r$ alleles at a single locus divide into two groupings (all genotypes $\mathscr{C}_{m}$ become males unambiguously, while all genotype zygotes of $\mathscr{C}_{f}$ become females). Assuming random mating in this population, the only stable equilibria (or equilibrium surfaces) entail one-to-one sex ratio, i.e., the frequency of a stable equilibrium phenotype has $\operatorname{freq}\left(\mathscr{C}_{m}\right)=$ $\operatorname{freq}\left(\mathscr{C}_{f}\right)=1 / 2$.

It should be emphasized that with probabilistic $\left(0<m_{i j}<1\right)$ sex determination, non-even-sex ratio stable equilibrium states are possible. This perhaps makes sense if the probabilistic mechanism is associated with endogenous or exogenous genetic and environmental covariates. 
TABLE 7.1. Comparisons Between the Occurrences of Symmetric Versus Even-Sex Ratio Equilibrium States

\begin{tabular}{llllll}
\hline Number of alleles & 2 & $3^{\dagger}$ & $4^{\dagger}$ & $5^{\dagger}$ & $6^{\dagger}$ \\
\hline Convergence to $1: 1$ sex ratio & & .69 & .83 & .90 & .93 \\
\hline Matrices leading to : & & & & & \\
(i) $1: 1$ sex ratio only & .39 & .55 & .72 & .74 & .85 \\
(ii) symmetric equilibria only & .36 & .17 & .06 & .02 & .02 \\
(iii) either & .25 & .28 & .22 & .24 & .13 \\
\hline
\end{tabular}

$\dagger$ Numerical simulations from 200 random sex-determination matrices with 10 random starting points for each.

TABLE 7.2. Comparisons Between the Rates of Convergence*

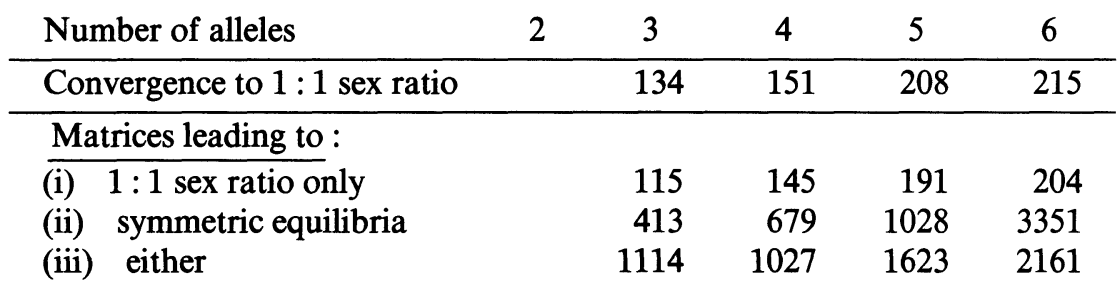

*Average numbers of generations for attainment of an equilibrium state. The iterations on (7.2) were terminated when no change in the sixth decimal place occurs for two successive generations.

The relative occurrence of symmetric (non one-to-one) versus even-sex ratio equilibria. In order to contrast the extent of stability for symmetric versus even-sex ratio equilibrium states, we constructed 200 random sex-determination matrices (each entry independently uniformly distributed on [0,1]). In each case we iterated the transformation (7.2) until an equilibrium was attained. The computer output is recorded in Tables 7.1 and 7.2.

As the number of alleles involved increases, even-sex ratio equilibrium surfaces are more likely to occur and their numbers to increase. It is worth noting that convergence to a one-to-one sex ratio is, in general, faster than convergence to a symmetric equilibrium (from three to ten times in our simulations). The difference is accented with more alleles.

Optimality of one-to-one sex ratio realizations. The property of ESS (evolutionary stable strategy) introduced by Maynard Smith and Price (1973) has been cogently applied in many studies of behavioral genetic models including sex ratio evolution (Charnov (1982)), genetics of altruism (Charlesworth (1978)), optimal dispersal rates (Motro (1982)) and in models of frequency dependent selection (Lessard (1984)). See also Zeeman $(1980,1981)$. It is widely conjectured that an even-sex ratio is optimal “ESS" (see §5). In the framework of the 
sex-determination model of $r$-alleles $((7.2))$, the optimality property is understood to mean that with the introduction of a new allele from $r$ to $r+1$ alleles, all possible stable equilibrium states for the extended model cannot attain a sex ratio farther from one-to-one than exists in the $r$-allele subsystem. This expresses an evolutionary tendency toward an even-sex ratio.

In this framework, we consider a symmetric equilibrium $\left\{\mathbf{p}^{*}, \mathbf{p}^{*}\right\}, p_{i}^{*}>0$, $i=1, \ldots, r$, for the $r$-allele model with $w^{*}=\left\langle M \mathbf{p}^{*}, \mathbf{p}^{*}\right\rangle<1 / 2$. With a new allele $\mathrm{A}_{r+1}$, let $m_{i, r+1}$ be the probability for a zygote of genotype $\mathrm{A}_{i} \mathrm{~A}_{r+1}$ to be a male and $\left(1-m_{i, r+1}\right)$ to be a female, respectively. The marginal fraction of male progeny carrying allele $\mathrm{A}_{r+1}$ at the equilibrium state $\mathbf{p}^{*}$ is $w_{r+1}^{*}=$ $\sum_{i=1}^{r} p_{i}^{*} m_{i, r+1}$. A symmetric equilibrium $\left\{\mathbf{p}^{*}, \mathbf{p}^{*}\right\}$ stable for the $r$-allele sex-determination model becomes unstable with the introduction of $\mathrm{A}_{r+1}$ if and only if

$$
w_{r+1}^{*}>w^{*} \quad \text { provided } w^{*}<1 / 2 .
$$

(The condition for local instability at $\mathbf{p}^{*}$ is $w_{r+1}^{*}<w^{*}$ if $w^{*}>1 / 2$.) These conditions do not require that the marginal sex ratio $w_{r+1}^{*}$ at $\mathbf{p}^{*}$ should be closer to $1 / 2$ than $w^{*}$. However, in case of instability, it was conjectured that the augmented system in the long run would attain a sex ratio closer to one-to-one than existing at the previous equilibrium.

We can prove

THEOREM 7.5. Let $\mathbf{p}^{*}$ be a stable symmetric equilibrium of (7.2) with sex ratio $w^{*}=\left\langle\mathbf{p}^{*}, M \mathbf{p}^{*}\right\rangle$ which becomes unstable following the introduction of a new allele $\mathrm{A}_{r+1}$. Then, for the augmented $(r+1)$-allele system with genericy conditions (Definition 7.1) in force, either: (i) there exists a unique stable symmetric equilibrium whose sex ratio is closer to $1 / 2$ compared to $w^{*}$ and which does not coexist with any even-sex ratio equilibrium, or (ii) $\left\{\mathbf{p}^{*}, \mathbf{p}^{*}\right\}$ is enclosed by a pair of even-sex ratio equilibrium surfaces containing no stable symmetric equilibria.

Under the conditions of the above theorem, the only stable equilibrium points attainable from $\mathbf{p}^{*}$ entail sex ratio closer (compared to $w^{*}$ ) to $1 / 2$ or equal to $1 / 2$.

Part (ii) has independent interest from an evolutionary perspective if it is assumed that new alleles are introduced singly. The proof decisively relies on the fact that $w(\mathrm{p})$ is a quadratic form.

Theorem 7.5 proposes that if $\mathbf{p}^{*}$ is unstable, then ultimately an equilibrium state will be reached closer (compared to $w^{*}$ ) to $1 / 2$ or equal to $1 / 2$. We can also interpret Theorem 7.5 as expressing an "optimality" property in favor of an even-sex ratio determination. On the basis of this result we surmise that with increasing allelism the population sex ratio is inclined toward an even-sex ratio. Any perturbations on a sex ratio equilibrium state render it unstable, ultimately achieve a sex ratio closer to $1 / 2$. Moreover, an even-sex ratio equilibrium persists as stable with the introduction of any additional allele involved in sex determination.

Although the predicted evolutionary sex ratio, by virtue of Theorem 7.5, tends toward one-to-one, nevertheless, for any finite allele sex-determination model as in (7.2), there can coexist a stable symmetric equilibrium with biased sex ratio and a stable surface of even-sex ratio equilibria. 
MODEL II. Multiallelic sex ratio determination model under maternal (or paternal) genotypic control. In concluding this section it is illuminating to briefly consider the model where sex ratio determination depends on the maternal genotype.

Consider a dioecious population with a sex-determining mechanism entailing $r$ alleles $A_{1}, A_{2}, \ldots, A_{r}$ where the sex of the offspring is governed by its maternal genotype. Documentation and interpretations of this mechanism are amply illustrated in Charnov (1982). In the present context we prescribe $m_{i j}$ as the expected proportion of males in the progeny of an $\mathrm{A}_{i} \mathrm{~A}_{j}$ female parent. The symmetric matrix $M=\left\|m_{i j}\right\|_{i}^{r}$ will be called the maternal sex ratio determination coefficient matrix.

Probabilistic sex determination with $0<m_{i j}<1$, subject to mother control can result from influences of modifier genes with interactions to the cytoplasmic millieux. The maternal physiological capabilities and neonatal responses to environmental conditions can be contributory factors. The maternal control can be latent or facultative.

Let $\tilde{p}_{i j}$ and $\tilde{q}_{i j}$ be the frequency of the ordered $\mathrm{A}_{i} \mathrm{~A}_{j}$ genotypes (the first allele $A_{i}$ deriving from the maternal side and $A_{j}$ from the paternal side) in the female and male populations, respectively. The corresponding frequencies of the unordered $\mathrm{A}_{i} \mathrm{~A}_{j}$ genotype are $2 p_{i j}=\tilde{p}_{i j}+\tilde{p}_{j i}$ and $2 q_{i j}=\tilde{q}_{i j}+\tilde{q}_{j i}$ for $i \neq j$ ( $p_{i i}$ and $q_{i i}$ when $i=j$ ).

An $A_{i}$ gamete is contributed from a maternal parent of genotype $A_{i} A_{l}$ with probability $\left(\tilde{p}_{i l}+\tilde{p}_{l i}\right) / 2=p_{l i}$. Note that $\frac{1}{2} \sum_{j}\left(\tilde{p}_{j k}+\tilde{p}_{k j}\right)=p_{k}, k=1, \ldots, r$, is the $k$ th allele frequency in the female population and the corresponding male allele frequency is $\frac{1}{2} \sum_{l}\left(\tilde{q}_{k l}+\tilde{q}_{l k}\right)=q_{k}$.

Under random mating the expected fraction of male offspring in the next generation with sex determination subject to mother control as delineated above is

$$
\tilde{q}_{i j}^{\prime}=\sum_{k} \sum_{l} m_{i l} \frac{\tilde{p}_{i l}+\tilde{p}_{l i}}{2} \frac{\tilde{q}_{j k}+\tilde{q}_{k j}}{2} .
$$

It is convenient to introduce the quantities $z_{i}=\sum_{l} m_{i l} p_{i l}, i=1, \ldots, r$, such that $z_{i} / p_{i}, i=1, \ldots, r$, can be interpreted as the probabilities that an offspring receiving a maternal allele $\mathrm{A}_{i}$ is a male. Obviously,

$$
w=\sum_{i=1}^{r} z_{i}=\sum_{i, l} m_{i l} p_{i l}
$$

is the sex ratio of the next generation.

The recursion equations can be compactly written in the form

$$
q_{i j}^{\prime}=\frac{\tilde{q}_{i j}^{\prime}+\tilde{q}_{j i}^{\prime}}{2}=\frac{z_{i} q_{j}+z_{j} q_{i}}{2 w}
$$

and

$$
p_{i j}^{\prime}=\frac{\left(p_{i}-z_{i}\right) q_{j}+\left(p_{j}-z_{j}\right) q_{i}}{2(1-w)}
$$


The above system can be reduced to the component variables of $\mathbf{p}, \mathbf{q}$, and $\mathbf{z}$, since only these variables appear on the right of (7.15), leading to

$$
\begin{gathered}
\mathbf{q}^{\prime}=\mathbf{q} / 2+\mathbf{z} / 2 w \\
\mathbf{p}^{\prime}=\mathbf{q} / 2+(\mathbf{p}-\mathbf{z}) / 2(1-w) \\
\mathbf{z}^{\prime}=\frac{(\mathbf{p}-\mathbf{z}) \circ M \mathbf{q}+\mathbf{q} \circ M(\mathbf{p}-\mathbf{z})}{2(1-w)}
\end{gathered}
$$

with $w=\sum_{i=1}^{r} z_{i}$.

At equilibrium it is easy to deduce the relationships

$$
w(1-2 w) \mathbf{p}=(1-2 w) \mathbf{z}, \quad \mathbf{q}=\mathbf{z} / w .
$$

Thus, at equilibrium either $w=1 / 2$ or $\mathbf{z}=w \mathbf{p}$, and then $\mathbf{p}=\mathbf{q}$. As in Model I, there are two equilibrium types, the symmetric equilibria and the even-sex ratio equilibria having $w=1 / 2$. For a symmetric equilibrium state $\left\{\mathbf{q}^{*}, \mathbf{p}^{*}, \mathbf{z}^{*}\right\}$ with $\mathbf{p}^{*}=\mathbf{q}^{*}, \mathbf{z}^{*}=w^{*} \mathbf{q}^{*}$, the recursion (7.16) reduces to

$$
\mathbf{p}^{\prime}=(\mathbf{p} \circ M \mathbf{p}) /\langle\mathbf{p}, M \mathbf{p}\rangle
$$

as in the classical one-locus viability system, and $\mathbf{p}^{*}$ is an equilibrium for the one-locus viability recursion (6.4).

For a nonsymmetric (even-sex ratio) equilibrium (confining attention to the symmetrically generic case, i.e., $\tilde{\mathbf{p}} \neq \tilde{\mathbf{q}}$ and $\tilde{w}=1 / 2$ ), a little manipulation of equation (7.16) gives $\tilde{\mathbf{q}}=B(\tilde{\mathbf{p}}-\tilde{\mathbf{z}}) \tilde{\mathbf{q}}$, where the matrix $B(\mathbf{p})$ is defined as in (7.6), so $\tilde{\mathbf{q}}=2 \tilde{\mathbf{z}}$ is the principal right eigenvector of $B(\tilde{\mathbf{p}}-\tilde{\mathbf{z}})$ of eigenvalue 1 . Setting $\mathbf{t}=2(\tilde{\mathbf{p}}-\tilde{\mathbf{z}}), \mathbf{t}$ is a frequency vector for which $\tilde{\mathbf{q}}$ is a right eigenvector of eigenvalue 1 of $\boldsymbol{B}(\mathbf{t})$ and $\tilde{\mathbf{q}}=2 \tilde{\mathbf{z}}$. The above discussion can be summarized in the following statement.

THEOREM 7.6. The maternal control sex ratio determination model (7.16) with $M$ symmetric generic (in the sense of Definition 7.1) admits as symmetric equilibria the equilibria of the one-locus viability model with matrix $M$, and as even-sex ratio equilibria, $\{\tilde{\mathbf{p}}, \tilde{\mathbf{q}}, \tilde{\mathbf{z}}\}$ with $\tilde{\mathbf{z}}$ corresponding to the eigenvector $\rho(B(\mathbf{s}))$ $=1, \mathbf{s}=2(\tilde{\mathbf{p}}-\tilde{\mathbf{z}})$, where $\rho(B(\mathbf{s}))$ is the spectral radius of $B(\mathbf{s})=D_{M \mathbf{s}}+\mathbf{s} \circ M$ defined on $\Delta$ and $\tilde{\mathbf{q}}=2 \tilde{\mathbf{z}}$.

Thus to find an even-sex ratio equilibrium we determine a frequency vector $\tilde{\mathbf{s}}$ for which $\rho(B(\tilde{\mathbf{s}}))=1$. Let its principal right eigenvector be $\tilde{\mathbf{q}}$. Then $\tilde{\mathbf{q}} / 2=\tilde{\mathbf{z}}$ and $\tilde{\mathbf{p}}=\tilde{\mathbf{s}} / 2+\tilde{\mathbf{z}}$, providing $\{\tilde{\mathbf{q}}, \tilde{\mathbf{p}}, \tilde{\mathbf{z}}\}$.

The characterizations of the equilibrium forms for maternal sex determination and transformation equations (7.16) are akin to those described in relation to model (7.2).

We formally state the results:

THEOREM 7.7. (i) The maternal control sex ratio determination model (7.16) with $M$ symmetrically generic (in the sense of Definition 7.1) admits as symmetric equilibria the equilibria of the one-locus viability model with matrix $M$, and as even-sex ratio equilibria $\{\tilde{\mathbf{p}}, \tilde{\mathbf{q}}, \tilde{\mathbf{z}}\}$ as determined from the matrix $B(\mathbf{s})$ described after Theorem 7.6. 
(ii) A symmetric equilibrium $\left\{\mathbf{p}^{*}, \mathbf{q}^{*}, \mathbf{z}^{*}\right\}, \mathbf{z}^{*}=w^{*} \mathbf{p}^{*}, \mathbf{p}^{*}=\mathbf{q}^{*}$ with $w^{*}<\frac{1}{2}$ $\left(w^{*}>\frac{1}{2}\right)$ is stable if and only if $\mathbf{p}^{*}$ is stable for the one-locus viability model of viability matrix $M(U-M)$.

(iii) If an even-sex ratio equilibrium exists, it is embedded in an $(r-1)$ dimensional surface of even-sex ratio equilibria.

(iv) Stable symmetric and even-sex ratio equilibria cannot coexist segregating the same alleles.

(v) The only stable equilibria resulting with the introduction of a mutant allele have a stable sex ratio representation the same or closer to one-to-one. The symmetric equilibria are the same for zygotic or maternal genotypic sex ratio determination but the rates of convergence to the stable equilibria generally differ.

We conclude this section with some concrete examples of the equilibrium realizations to the sex-determination model (7.2). Analogous results are available under maternal control sex determination and haplodiploid systems.

$A$ complete enumeration of the stable equilibrium states of genotype sex ratio determination involving $r=2$ alleles. The model of sex ratio determination of $\$ 2$ involving $r=2$ alleles was studied in Eshel (1975). The parameterization is

$$
M=\left(\begin{array}{cc}
\alpha & \beta \\
\beta & \gamma
\end{array}\right), \quad \begin{aligned}
& \alpha=\text { probability that an } \mathrm{A}_{1} \mathrm{~A}_{1} \text { zygote is male } \\
& \beta=\text { probability that an } \mathrm{A}_{1} \mathrm{~A}_{2} \text { zygote is male } \\
& \gamma=\text { probability that an } \mathrm{A}_{2} \mathrm{~A}_{2} \text { zygote is male }
\end{aligned}
$$

The transformation equations (7.2), in this case setting $q_{1}=q, p_{1}=p$, reduce to

$$
\begin{aligned}
& q^{\prime}=\frac{1}{2 w}[2(\alpha-\beta) p q+\beta(p+q)] \\
& p^{\prime}=\frac{1}{2(1-w)}[2(\beta-\alpha) p q+(1-\beta)(p+q)]
\end{aligned}
$$

and $w=(\alpha-2 \beta+\gamma) p q+(\beta-\gamma)(p+q)+\gamma$.

We provide an exhaustive accounting of the equilibrium alternatives for this model summarized in Table 7.3. The validation of global stability derives from the monotonicity endowments (increasing in each of the variables $p$ and $q$ of the transformation equations (7.19)).

\section{TABLE 7.3. Stable Equilibrium Alternatives for $1 / 2<\beta<1$}

(When $\beta<1 / 2$ the results are identical with $\alpha, \beta$ and $\gamma$ replaced by $1-\alpha$, $1-\beta$, and $1-\gamma$, respectively.) The symmetric equilibrium $p^{*}$ when extant has the formula $q^{*}=p^{*}=(\beta-\gamma) /(2 \beta-\alpha-\gamma)$. The two possible even-sex ratio equilibria are denoted by $\{\tilde{q}, \tilde{p}\}$ and $\{\tilde{\tilde{q}}, \tilde{\tilde{p}}\}$. These are obtained as the solutions $\tilde{p}$ of the quadratic

$$
\begin{aligned}
& x^{2}[\beta(\alpha+\gamma-2 \beta)-2(\beta-\gamma)(\alpha-\beta)] \\
& \quad+x[(\beta-\gamma)+(\alpha-\beta)(1-2 \gamma)]+((1-\beta) / 2)(2 \gamma-1)=0
\end{aligned}
$$

with $\tilde{q}=(\tilde{p} \beta) /(1-\beta-2(\alpha-\beta) \tilde{p})$. When two unsymmetric equilibria exist, we label them by ordering $\tilde{q}<\tilde{\tilde{q}}$. If a single nonsymmetric equilibrium exists, we denote it by $\{\tilde{q}, \tilde{p}\}$. 
TABLE 7.3 (continued)

\begin{tabular}{|c|l|l|}
\hline Parameter Description & \multicolumn{1}{|c|}{ Stable Equilibrium } & Domains of Attractions \\
\hline I: $\quad \beta<\gamma<1,0<\alpha<\frac{1}{2}$ & $\begin{array}{l}\text { A unique even-sex ratio } \\
\{\tilde{q}, \tilde{p}\} \text { exists }\end{array}$ & globally stable \\
\hline II: $\quad \beta<\gamma<1, \frac{1}{2}<\alpha<\beta$ & $\begin{array}{l}\text { Even-sex ratio equli- } \\
\text { bria do not exist: the } \\
\text { pure symmetric state } \\
\{1,1\} \text { is uniquely stable }\end{array}$ & globally stable \\
\hline III: $\quad \beta<\gamma<1, \beta<\alpha<1$ & $\left\{p^{*}, p^{*}\right\}$ uniquely stable & globally stable \\
\hline IV: $\quad \frac{1}{2}<\gamma<\beta, 0<\alpha<\frac{1}{2}$ & $\{0,0\}$ and $\{\tilde{q}, \tilde{p}\}$ stable & $\begin{array}{l}\left\{p^{*}, p^{*}\right\} \text { exists but is } \\
\text { unstable; } 0<p^{*}<\tilde{q}\end{array}$ \\
\hline V: $\quad \frac{1}{2}<\alpha<\beta, \frac{1}{2}<\gamma<\beta$ & $\begin{array}{l}\text { Both corner symmetric } \\
\text { equilibria }\{0,0\} \text { and } \\
\{1,1\} \text { are stable }\end{array}$ & $\begin{array}{l}\left\{p^{*}, p^{*}\right\} \text { exists but is } \\
\text { unstable. }\end{array}$ \\
\hline VI: $\quad \frac{1}{2}<\gamma<\beta, \beta<\alpha<1$ & $\{0,0\}$ uniquely stable & globally stable \\
\hline VII: $\quad \gamma<\frac{1}{2}, \alpha<\frac{1}{2}$, & $\begin{array}{l}\{\tilde{q}, \tilde{p}\} \text { and }\{\tilde{q}, \tilde{p}\} \text { are } \\
\text { both stable }\end{array}$ & $\begin{array}{l}\left\{p^{*}, p^{*}\right\} \text { exists and is } \\
\text { unstable; } \tilde{q}<p^{*}<\tilde{q}\end{array}$ \\
\hline VIII: $\quad 0<\gamma<\frac{1}{2}, \frac{1}{2}<\alpha<\beta$ & $\begin{array}{l}\{1,1\} \text { and }\{\tilde{q}, \tilde{p}\} \text { are } \\
\text { stable }\end{array}$ & $\begin{array}{l}\left\{p^{*}, p^{*}\right\} \text { exists; } \\
\tilde{q}<p^{*}<1\end{array}$ \\
\hline IX: $\quad 0<\gamma<\frac{1}{2}, \beta<\alpha<1$ & $\{\tilde{q}, \tilde{p}\}$ uniquely stable & globally stable \\
\hline X: $\quad \alpha<\frac{1}{2}, \gamma<\frac{1}{2}$, & $\left\{p^{*}, p^{*}\right\}$ uniquely stable & globally stable \\
\hline$w^{*}<\frac{1}{2}$ & & \\
\hline
\end{tabular}

(The parameter $w^{*}=\left(\alpha \gamma-\beta^{2}\right) /(\alpha+\gamma-2 \beta)$.)

\section{Quantitative models of sex ratio theory (environmental sex determination).} Bulmer and Bull (1982) introduced a series of models with sex determination responding to environmental conditions interacting with many genes of small effects. They note that among several reptile species (e.g., crocodiles and especially turtles; see Bull (1981) and Charnov (1982, Chapter 11)) the sex of an offspring may depend crucially on incubation temperature. Other environmental sex determinants (ESD) can be related to prenatal or neonatal birth size, physiological conditions, resource availabilities of host and other ecological environmental factors.

Consider a population in which the sex is determined (at conception) in the zygote through multifactorial effects. The simplest model of this kind is to assume that the sex response trait is governed by a continuous phenotype variable $X$ (e.g., size, food resources, degree of exposure to sunlight), such that an individual $X=x$ becomes a male with probability $p(x)$, and a female with probability $1-p(x)$. For the special case

$$
p(x)= \begin{cases}1, & x>v \\ 0, & x \leqslant v\end{cases}
$$


the sex response in favor of maleness versus femaleness depends on the phenotype value exceeding the threshold level $v$ or not. Let the population distribution of the phenotype values at the current generation be $f(x)$, i.e., the fraction of the population of trait value between $(x, x+\Delta x)$ is approximately $f(x) \Delta x$. The sex ratio at conception is

$$
r=p(x) f(x) d x .
$$

The density of phenotypes in the male population is

$$
f^{*}(x)=p(x) f(x) / r,
$$

and that of females

$$
f^{* *}(x)=[1-p(x)] f(x) /(1-r) .
$$

For compactness, we denote the associated random variables as $X^{*}$ and $X^{* *}$, respectively. At the adult stage the proportions of males and females can be adjusted in several ways. We will focus in the present discussion on the following case. At mating, that is, as adults, the sex ratio of males to females equalizes with trait distribution that of (8.3).

We assume random mating with the offspring phenotypic expression a blend of the parental trait values plus an individual residual addend. More explicitly, the random variable of the offspring $X^{*}$ is taken to be

$$
X=h \frac{X^{*}+X^{* *}}{2}+\xi
$$

where $h$ can be construed as a heritability factor, and $\xi$ is an independent environmental contribution to the trait value governed by a density function $e(x)$ independent of the generation epoch.

More general formulations allow $p(x)$ to vary systematically or stochastically in time and/or space where also the perturbation terms $\xi$ can be taken as a random process involving some dependence over time.

For the analysis in this paper we focus on the case of (8.4a) with $h=1$, $\xi=0$ so that the offspring trait value is $\tilde{X}$ and

$$
\tilde{X}=\left(X^{*}+X^{* *}\right) / 2 \text {. }
$$

The mean value of $\tilde{X}$ is

$$
\tilde{m}=m+\frac{1-2 r}{2 r(1-r)} \int(x-m) p(x) f(x) d x .
$$

By virtue of the Tchebycheff rearrangement inequality (since $p(x)$ and $x-m$ are both increasing functions of $x$ ) we have

$$
\int(x-m) p(x) f(x) d x \geqslant\left(\int p(x) f(x) d x\right)\left(\int(x-m) f(x) d x\right)=0,
$$

and therefore,

$$
\begin{array}{ll}
\tilde{m}-m>0 & \text { when } r<1 / 2, \\
\tilde{m}-m<0 & \text { when } r>1 / 2 .
\end{array}
$$

We could expect that the mean $m$ over successive generations tends monotonically to $1 / 2$. This is generally not true. 
The distribution of $\tilde{X}$ is

$$
\tilde{f}(x)=2 \int_{-\infty}^{\infty} f^{*}(\xi) f^{* *}(2 x-\xi) d x .
$$

Comparisons of sex ratio over two successive generations. For the sex threshold model (8.1) with threshold level $v$ and transformation relation $(8.4 \mathrm{~b})$, where the total population phenotype density is $f(x)$, the sex ratio of the following generation is

$$
\begin{aligned}
r^{\prime} & =\operatorname{Pr}\left\{\frac{X^{*}+X^{* *}}{2}>v\right\}=\int_{-\infty}^{v} \frac{f(x \xi)}{F(v)}\left(\frac{\int_{2 v-\xi}^{\infty} f(\eta) d \eta}{1-F(v)}\right) d \xi \\
& =\frac{\int_{v}^{\infty} f(2 v-\xi)[1-F(\xi)] d \xi}{F(v)[1-F(v)]} .
\end{aligned}
$$

We investigate the question: For $r<1 / 2$ under what conditions do the relations

$$
r^{\prime}>r \text { and } r^{\prime}<1 / 2
$$

hold? and, of course, the reverse of these.

These questions motivate a number of interesting comparison inequalities for random variables. We have

THEOREM 8.1. If $f(x)$ is a symmetric log-concave density then $r^{\prime}<1 / 2$ for $v>0$ and $r^{\prime}>1 / 2$ for $v<0$.

This theorem relies on the fact (Proschan (1965); see also Karlin (1968, p. 384)) that for $f$ a symmetric log-concave density,

$$
\left(Z_{1}+Z_{2}\right) / 2 \text { is more peaked than } Z \text {, }
$$

meaning that

$$
\int_{0}^{a} f_{(2)}(\xi) d \xi>\int_{0}^{a} f(\xi) d \xi \text { for all } a>0
$$

where

$$
f_{(2)}(v)=2 \int_{-\infty}^{\infty} f(\xi) f(2 v-\xi) d \xi
$$

is the density of $\left(Z_{1}+Z_{2}\right) / 2$, each $Z_{i}$ independently distributed following the density $f(\xi)$.

The result of Theorem 8.1 is not correct for a general even density.

THEOREM 8.2. Let $X$ be a symmetric random variable with density $f$. Let $X_{1}$, $X_{2}, Z_{1}, Z_{2}, Z_{3}$ be independent realizations based on $f$.

If

$$
\operatorname{median}\left(Z_{1}, Z_{2}, Z_{3}\right) \stackrel{\text { more peaked }}{\succ}\left(X_{1}+X_{2}\right) / 2
$$

then for $r<1 / 2$, we have $r^{\prime}>r$. A sufficient condition that (8.10) holds is that $f(\xi)$ be an even-P $F_{\infty}$ density (i.e., $f(x)$ has a Laplace transform $\phi(z)$ such that

$$
\frac{1}{\phi(z)}=e^{-\gamma z^{2}} \prod_{i=1}^{\infty}\left(1+a_{i} z\right) e^{-a_{i} z}
$$


with $\gamma \geqslant 0, a_{i}$ real, and $\sum_{i=1}^{\infty} a_{i}^{2}<\infty$ so that $\phi(z)^{-1}$ is an entire function of order 2 of Pólya-Laguerre type; see Karlin (1968, Chapter 7)).

The class of $\mathrm{PF}_{\infty}$ densities includes the normal density, the double exponential density $f(x)=e^{-|x|}$ and all convolutions of translation and scale changes of these special densities.

REMARK. Inequality (8.10) is not correct for arbitrary symmetric log-concave densities. For example, take $f(\xi)=C e^{-|\xi|^{\alpha}}, C^{-1}=\int_{-\infty}^{\infty} e^{-|\eta|^{\alpha}} d \eta$, which is log-concave for all $\alpha \geqslant 1$. For $\alpha$ large enough (even $\alpha \geqslant 4$ ) (8.10) cannot hold.

In view of (8.10) the peakedness comparison of the densities of median $\left(X_{1}, \ldots, X_{2 n-1}\right)$ versus $\left(X_{1}+\cdots+X_{n}\right) / n$ is of interest. We have the following by-product of mathematical and statistical interest emanating from our studies of quantitative sex ratio evolution.

THEOREM 8.3. If $f(x)$ is a symmetric $P F_{\infty}$ density, then

$$
\operatorname{median}\left(X_{1}, X_{2}, \ldots, X_{2 n-1}\right) \text { is more peaked than } \frac{X_{1}+\cdots+X_{n}}{n}
$$

where $X_{i}$ are independent identically distributed with density $f(x)$.

In particular, for $2 n-1$ independent observations from a normal density of mean zero, the density of median $\left(X_{1}, \ldots, X_{2 n-1}\right)$ is more peaked than the density of $\left(X_{1}+\cdots+X_{n}\right) / n$.

We conclude with a very general theorem on the evolution of the phenotype variable underlying sex expression governed by the equation

$$
X_{t+1}=\left(X_{t}^{*}+X_{t}^{* *}\right) / 2,
$$

where $X_{t}^{*}\left(X_{t}^{* *}\right)$ is the male (female) adult sex ratio of generation $t$, and $X_{t+1}$ the offspring value. Assume the threshold ((8.1)) criterion underlying sex expression.

THEOREM 8.4. If $f(x)$ is a log-concave density then $\operatorname{Var} X_{t} \rightarrow 0$ at a geometric rate and the sex ratio $r_{t}$ approaches $1 / 2$.

The proof of Theorem 8.4 uses the result that if $U$ is a real random variable having a log-concave density, then the conditional variances $\operatorname{Var}[U \mid U \leqslant b]$ and $\operatorname{Var}[U \mid U \geqslant a]$ are decreasing as $a$ increases and $b$ decreases (Karlin (1982a)).

The convergence analyses of the transformation equations

$$
X_{t+1}=\alpha X_{t}^{*}+\beta X_{t}^{* *}+\xi_{t}
$$

are also accessible. Detailed analyses of the above recursion system and further studies of polygenic sex ratio models under mother control and for haplodiploid organisms will be presented elsewhere (Karlin and Lessard (1984)).

9. Perspectives and future prospects of mathematical evolutionary theory. This section starts with a brief discussion of how evolutionary biology differs from physics in concepts, issues, and methodology. Several surprising discoveries emanating from the genetics of the past decade are noted. We then highlight a number of prominent current research areas in quantitative evolutionary theory, and consider the kinds of mathematical and statistical analyses 
that can play an important role in dealing with these areas. In all likelihood the new genetics emanating from the recombinant technology, like the genetics of the past (see §3), will lead to new interesting mathematical structures that will also be of value to other sciences.

Evolutionary biology and physics. A main goal of physical theory is to delineate a set of basic laws that provide a coherent description of the forces acting on matter, and their consequences. Physicists see these laws as being comparatively few; their main effort is to synthesize rather than classify. According to Feynman (1964, Vol. 1, Chapter 38), "The most dramatic moments in the development of physics are those in which great syntheses take place, where phenomena which appeared to be different are discovered to be the same. The history of physics is the history of such syntheses and the bases of success of physical science is that we are able to synthesize."

Many physicists and chemists would contend that from understanding the properties of particles responding to various forces and interactions, we will know the basic principles governing all phenomena. For example, Eigen (1971) states: "If we want to close the gap between physics and biology we have to find out what selection means in precise molecular terms which can ultimately be described in quantum mechanical theory. We have to derive Darwin's principles from known properties of matter." This reductionist viewpoint is inappropriate to evolutionary theory and most of organismal biology for reasons implicit in our later discussions.

Biological phenomena divide into two main categories depending on the unit under study. The first category, organismal biology, includes the fields of evolution, ecology, ethology, biogeography, systematics, paleontology, and the new sociobiology. Possible units of study are the individual, a population, a species, an ecosystem, and more complex organizational forms. A population or an ecosystem cannot be summarized by simply listing its component parts; also entailed are the many complex interactions between parts.

The second category, molecular biology, encompasses molecular genetics, biochemistry, biophysics, and a myriad of specialties such as immunology, morphogenesis, cytology, and embryology. Typical areas of study are biological molecules, macromolecules, cell constituents, enzyme processes, regulation mechanisms and energy systems.

The levels of biological processes range from cell component mechanisms, to the complexity of multicellular workings, to the whole organisms, to the relations of individuals within and between populations, to the organizational structure of ecosystems, and beyond. Perhaps for anthropomorphic reasons this hierarchy is usually seen as culminating with man, as distinguished by self-awareness and the ability within limits to control his own destiny. Biological structures involve elements and functions of life processes from the simple units of DNA replication, transcription, and translation to the complex attributes of cognition, learning, purpose, and behavior. Diversity and complexity in forms, mechanisms, and processes significantly increase with each level.

I will now suggest several comparisons between physics and evolutionary biology relative to methodologies and concepts. Although for convenience of 
exposition the discussion that follows tends to be dichotomized, in reality the differences discussed are ultimately of kind and degree.

The nature of the questions asked. Evolutionary theory tends to ask the questions why and what for in a nonteleological sense. For example, what maintains a species in a given environment? What value does a given behavior pattern provide a particular organism? Why do some populations vary more than others? What accounts for different life history strategies in form or frequency among similar species? Why do migration patterns differ significantly between two closely related bird populations?

Physics tends to ask the question how. How does the mechanism work? What are the basic particles, and how do they interact under different conditions? Physicists regard questions like "Why does matter exist?" as metaphysical. Even such a question as "Why are certain patterns mostly found and not others?" would be dispensed with by an appeal to stability considerations or minimum principles. Things just are the way they are; the interesting question is how they work. To be sure, there is evolution of a sort: stars are being born and going extinct, galaxies are changing, there is geological movement in plate tectonics. But this is very different from the dynamic biological evolution of our earth.

Asked "Are the laws of physics changing?", the physicist Dirac responded "No", with the qualification that the strength of electromagnetism may be changing. There is radioactive decay and changes among atomic and subatomic particles. This concept of change is very different from the biologist's.

Experiment and repeatability. Physicists constantly affirm that the ultimate test of all physical theory is observation. Eddington asserts that "qualitative theory is no theory", Lord Kelvin that qualitative theory is bad quantification. Qualitative theory may be the real (and paramount) stuff of ethology and sociobiology.

Physics generally aims for controlled experiments in which environmental and background conditions are judiciously manipulated. Such experiments are impossible in studying processes in vivo, and all but impossible in studying ecological or evolutionary phenomena. Even to describe the environment in a field study is a formidable, if not prohibitive, task. Various efforts to distinguish systematically between environments by using climatic parameters, demographic parameters, ecosystem dimensions, geological or chemical variables, life history attributes, etc. are often controversial.

Physics and chemistry experiments possess a substantial degree of repeatability, whereas biological observations (molecular biology excepted) are mostly nonrepeatable. Moreover, evolutionary events often involve significant historical (unique) factors that obviously vary over time. Populations come and go. No two individuals or populations are alike, and no individual in his life cycle remains the same. All organisms result from a blueprint of genetic information, but the program develops from interaction with environment. Where experiments are neither controllable nor repeatable, the doctrine of falsifiability, so important in physics, has little application.

Randomness and determinism. There is the old dictum of Laplace that randomness does not exist, it merely reflects our ignorance. Nevertheless, I 
would like to divide random phenomena into two types: randomness by design and randomness by complexity. In our discussion of evolutionary theory we contrasted the determinism of natural selection versus the randomness inherent to the events of mutation and recombination. Sexual crosses and incompatibilities in mating systems genuinely effect a shuffling of gene types. This is obviously a case of designed randomness acting by the evolutionary process on the biological systems. (The evolution of sex can perhaps be attributed to randomness by complexity; but having entered into the evolutionary dynamics, it now expresses randomness by design.) On the other hand, the interaction of genetic forces with the environment entails complex nonlinear effects with outcomes exhibiting irregular ergodic or chaotic behavior; and this appears to be randomness by complexity, the kind that Laplace probably had in mind. Mutation events may be of this kind.

The randomness of physical systems is mostly of the Laplace kind, i.e., randomness by complexity. The Fermi-Dirac and Boltzmann-Einstein statistics, based on the simple notion of throwing balls into boxes, serve only as theoretical models to study different conceptions of ensembles of particles.

Many different levels and forms of randomness are embedded in biological processes, among them degeneracies (e.g., genetic code), redundancies (e.g., multicopy DNA, multimeric proteins), and balance and flexibility in function (e.g., the induced-fit principle, the multitude of allozymes).

In physics, quantum theory sets forth a "probability language" designed to allow interpretations of mainly atomic or subatomic phenomena. This language is not consonant with that of mathematical probability theory. Probabilities for various states are not added directly; but rather the wave functions are added, reflecting the wave-particle dualism underlying interference patterns. Statistical mechanics uses the standard calculus of probabilities to describe the distributional properties of large numbers of colliding particles in various states, and transitions between them.

The uncertainty principle expresses an inherent fuzziness that must exist in any attempt to describe matter at the microscopic level. The fuzziness is mainly man-made, the result of disturbances imposed from outside the system. The uncertainty is embedded in the interference pattern induced by measurement with physical apparatus. It is not that physical processes are intrinsically fuzzy -in themselves they are completely determined by a full prescription of initial conditions-but rather that complete information to human observers is impossible. Since the possibility of precise measurement is even smaller in the biological and social sciences, we may legitimately call for the development of a (qualitative) statistical theory appropriate to those disciplines.

Laws, principles, theorems. The enunciation of physical laws is at the core of the physical sciences. Physical theory constantly strives to attain an economy of principles and fundamental entities. By contrast there are virtually no theorems for evolutionary or ecological phenomena, since exceptions are ever-present. There are, to be sure, universals (e.g., Mendelism, the DNA-RNA protein complex, recombination), but they are characterized by enormous variability. There are few unique spatial and temporal stable frequency patterns of alternative gene forms and species types; and at the same time there 
are many different possible stable states, such that which state occurs in a particular case may depend on historical factors or sampling fluctuation effects rather than deterministic selection forces.

A severe limitation in developing biological theory is that often we cannot begin to conceive of the array of alternatives. The present array of organisms resulting from DNA coding represents only a miniscule fraction of all possible organisms. Even for molecular biologists, who deal with problems seemingly more closely related to physical and chemical processes, the investigation of regulating and structural mechanisms when passing from viruses and bacteria to multicellular organisms involves coming to terms with far more possibilities than were contemplated two decades ago. These difficulties may be attributable partly to the intrinsic randomness of recombination and sex, and partly to the exceedingly complex nonlinearities embedded in the life process owing in part to changing environmental conditions.

Predictability and causality. Physics constantly strives for predictability. A classic example of excellent predictions without controlled experiments is the work of astronomers, and there have also been successful predictions of the existence of theretofore unknown subatomic particles, notably Dirac's prediction of antimatter and more recent predictions relating to meson particles. In history, by contrast, prediction is never feasible, although given a succession of historical events one can often explain the causal relationships between them. In a similar vein, evolutionists, ecologists, ethologists, and taxonomists tend to agree that prediction is rarely possible in their fields, even in a statistical sense. Theories in these fields are proposed strictly for the analysis of causes or for descriptive purposes.

The new genetics. The powerful new recombinant technology and the rapid DNA sequencing methodology developed over the past decade has brought us new perspectives on the nature and workings of genes. We were greatly surprised to learn of the existence of interrupted gene parts (exons-introns) having DNA segments that are transcribed but not translated into protein, the existence of mobile genes (transposons), the high order $\left(10^{6}\right)$ of repeated tandem DNA pieces, and the presence of thousands of direct repeats widely interspersed through the genome (the DNA content of all the chromosomes). A new dimension of variability involves restriction length fragment polymorphism (RLFP) in populations, i.e., variability with respect to the length of pieces where specific restriction enzymes cut DNA. Because RLFP are so widely distributed over the genome, these polymorphisms may help make it possible to identify the locations of important genes.

Another conundrum arising from the recombinant technology present in most higher organisms, as distinguished from viruses and bacteria, is the fact that $80 \%-90 \%$ of the genome is not transcribed and perhaps largely nonfunctional. We have still to assess the implications of these new findings for evolutionary theory.

Homologous recombination, involving the exchange of genetic material between homologous chromosomes at corresponding positions, is part of classical genetics. The recent discovery of abundant nonhomologous recombination is somewhat startling. Its mechanisms and implications are still being worked out. 
Another area of potential advance is the study of moderately large and large multigene families as distinguished from the study of unique genes. The component genes of a multigene family usually function approximately the same way as the family as a whole, but are differentially expressed in different life stages (e.g., as in the globin genes). An interesting class of multigene families is the immunoglobulin genes, which are fundamental in processes of immune response. The recombinant technology has significantly assisted in explaining how antibody diversity is generated.

Mathematics and the new genetics. Nucleic acid sequencing, engaged in by biochemists throughout the world, has been used to generate a data base currently totalling an estimated 2.5 million nucleotides. To search a large sequence or many smaller sequences and identify relevant features and structures, we need innovative computer programs coupled with rigorous statistical procedures. The problem of classifying patterns and homology relationships among DNA sequences within and between species is also worth the attention of mathematicians. The application of suitable mathematical techniques to the DNA data base may also help to resolve the old questions about the number of primordial genes.

The construction of evolutionary trees based on DNA sequences rather than protein sequences presents new opportunities and potentially new insights. The investigation of similarities and differences between individuals, populations, and species requires attention to genetic, environmental, and cultural factors. The issue and definition of species selection is relevant. The need for refined statistical procedures in this area is well recognized (see Nei and Roychoudbury (1982), Karlin et al. (1979)).

There are at least three forces that alter and shuffle DNA sequences: One is the process of homogenization (also called concerted evolution), which transforms certain DNA segments within and between chromosomes to the same condition as others. Another is the constantly occurring mutation events that create variation and speciation. The third, broadly speaking, is selection forces favoring certain types. Clearly, we need to develop quantitative models that accommodate the interplay of these forces.

Other problem areas that will need mathematical input are the classification of growth and extinction patterns, evolutionary rates, models for the description and classification of nonrandom mating dynamics, and the difficult problem of uncovering principles that may help explicate the nature and incidence of diversity and unity in biological systems.

Evolutionary theory is important to a proper understanding of living populations at all levels. Thus the analysis of gene frequency arrays assuredly has a part to play in anthropological comparisons and observations of animal behavior patterns that may lead to a better quantification of the genetics and environmental components of behavioral traits. Mathematics, as the language of quantitative measurement, is clearly central to these pursuits. The relevant mathematics undoubtedly requires hybridization of nonlinear analyses, compounded stochastic processes modeling, innovative statistical analysis of complex data, and the creative implementation of the gigantic computer methodology and all its ramifications. 


\section{REFERENCES}

G. BELL, 1982. The masterpiece of nature. The evolution and genetics of sexuality, Univ. of California Press, Berkeley, Calif.

F. BERNSTEIN, 1925. Zusammenfassende Betrachtungen über die erblichen Blutstrukturen des Menschen, Z. Abst. Vereb. 37, 237-269.

S. A. BooRman AND P. R. LEVITT, 1973. A frequency-dependent natural selection model for the evolution of social cooperation networks. Proc. Nat. Acad. Sci. USA 70, 187-189. 1980. The genetics of altruism, Academic Press, New York.

J. J. BuLl, 1981. Evolution of environmental sex determination from genotypic sex determination, Heredity 47, 173-184.

M. G. BULMER, 1980. The mathematical theory of quantitative genetics, Clarendon Press, Oxford.

M. G. BULMER AND J. J. Bull, 1982. Models in polygenic sex determination and sex ratio evolution, Evolution 36, 13-26.

B. Charlesworth, 1978. Some models of the evolution of altruistic behavior between siblings, J. Theoret. Biol. 72, 297-319.

E. L. Charnov, 1982. The theory of sex allocation, Princeton Univ. Press, Princeton, N. J.

B. C. Clarke, 1979. The evolution of genetic diversity, Proc. Roy. Soc. London Ser. A. 250, 543-574.

J. F. CROW AND M. KImURA, 1970. An introduction to population genetics theory, Harper and Row, New York.

Th. DobzhanSKy, 1951. Genetics and the origin of species, 3rd ed., Columbia Univ. Press, New York.

M. D. DonSKeR AND S. R. S. VARADHAN, 1975. On a variational formula for the principal eigenvalue for operators with maximum principle, Proc. Nat. Acad. Sci. U.S.A. 72, 780-783.

M. EIGEN, 1971. Self organization of matter and the evolution of biological macromolecules, Naturwissenschaften 58, 475-522.

J. A. ENDLER, 1977. Geographic variation speciation and clines, Princeton Univ. Press, Princeton, N. J.

I. ESHEL, 1972. On the neighbor effect and the evolution of altruistic traits, Theoret. Population Biol. 3, 258-277.

, 1975. Selection on sex ratio and the evolution of sex determination, Heredity 34, 351-361.

I. Eshel AND M. W. Feldman, 1982. On evolutionary genetic stability of the sex ratio, Theoret. Population Biol. 21, 430-439.

W. EWENS, 1972. The sampling theory of selectively neutral alleles, Theoret. Population Biol. 3, 87-112. ,1979. Mathematical population genetics, Springer-Verlag, Heidelberg.

W. Feller, 1951. Diffusion processes in genetics, Proc. 2nd Berkeley Sympos. on Math. Statist. and Probab. (J. Neyman, ed.), Univ. of California Press, Berkeley, Calif., pp. 227-246.

J. FelsensteIn, 1976. The theoretical population genetics of variable selection and migration, Ann. Rev. Genet. 10, 157-166.

R. P. FEYnMan, 1964. The Feynman lectures on physics, Vols. 1-3, Addison-Wesley, Reading, Mass.

R. A. FISHER, 1918. The correlation between relatives on the supposition of Mendelian inheritance, Trans. Roy. Soc. Edinburgh 52, 399-433.

1922. On the dominance ratio, Proc. Roy. Soc. Edinburgh 56, 321-341.

1930. The genetical theory of natural selection, Oxford Univ. Press, London (rev. ed., Dover)

W.'H. Fleming, 1975. A selection migration model in population genetics, J. Math. Biol. 2, 219-233.

S. FRIEDLAND AND S. KARLIN, 1975. Some inequalities for the spectral radius of nonnegative matrices and applications, Duke Math. J. 42, 459-490.

D. J. FUTUYMA, 1979. Evolutionary biology, Sinauer, Sunderland, Mass.

F. GALTON, 1889. Natural inheritance, Macmillan, London.

L. GinzBURG, 1979. Why are heterozygotes often superior in fitness, Theoret. Population Biol. 15, 264-267.

, 1983. Mathematical evolutionary theory, Benjamin, New York. 
J. B. S. Haldane, 1927. A mathematical theory of natural and artificial selection. V: Selection and mutation, Proc. Cambridge Philos. Soc. 23, 235-243.

1932. The causes of evolution, Harper \& Row, New York.

W. D. HAMILTON, 1964. The genetical evolution of social behavior. I, II, J. Theoret. Biol. 7, 1-52.

D. L. HARTL, 1980. Principles of population genetics, Sinauer, Sunderland, Mass.

P. W. HEDRICK, 1983. Genetics of populations, Van Nostrand Reinhold, New York.

P. W. Hedrick, M. E. Ginevan and E. P. EwING, 1976. Genetic polymorphism in heterogenous environments, Ann. Rev. Ecol. Syst. 7, 1-33.

P. W. HEDRICK, S. JAIN AND L. HOLDEN, 1978. Multilocus systems in evolution, Evolutionary Biology, Vol. 11, Plenum Press, New York.

S. KarıIN, 1968. Total positivity, Stanford Univ. Press, Stanford, Calif. , 1978. Theoretical aspects of multilocus selection balance. I, Studies in Mathematical Biology, Part II: Populations and Communities (S. A. Levin, ed.), MAA Studies in Math., Vol. 16, Math. Assoc. Amer., Wash., D. C., pp. 503-587. , 1979a. Principles of polymorphism and epistasis for multilocus systems, Proc. Nat. Acad. Sci. U.S.A. 76, 541-545. , 1979b. Models of multifactorial inheritance. I-IV, Theoret. Population Biol. 15, 308-438.

1982a. Some results on optimal partitioning of variance and monotonicity with truncation levels, Statistics and Probability: Essays in Honor of C. R. Rao (G. Kallianpur, P. R. Krishnaiah and J. K. Ghosh, eds.), North-Holland, Amsterdam, pp. 375-382. , 1982b. Classifications of selection-migration structures and conditions for a protected polymorphism, Evolutionary Biology, Vol. 14, Plenum Press, New York, pp. 61-204.

S. KARLIN AND H. AVNI, 1981. Analysis of central equilibria in multilocus systems: $A$ generalized symmetric viability regime, Theoret. Population Biol. 20, 241-280.

S. KARLin, R. KenetT AND B. Bonne-TAMiR, 1979. Analysis of biochemical data on Jewish populations. II: Results and interpretations of heterogeneity indices and distance measures with respect to standards, Amer. J. Human Genetics 31, 341-365.

S. KARLIN AND S. LeSSARD, 1983. On the optimal sex ratio, Proc. Nat. Acad. Sci. U.S.A. 180, 5931-5935.

1984. Theoretical studies of sex ratio evolution (to appear).

S. KARLIN AND C. MATESSI, 1983. Kin selection and altruism, Proc. Roy. Soc. London Ser. B 219, 327-353.

S. KARLIN AND H. M. TAYLOR, 1981. A second course in stochastic processes, Academic Press, New York.

M. KImURA, 1957. Some problems of stochastic processes in genetics, Ann. Math. Statist. 28, 882-901.

, 1964. Diffusion models in population genetics, J. Appl. Probab. 1, 177-232.

1983. The neutral theory of evolution, Cambridge Univ. Press, Cambridge.

M. KIMURA AND J. F. CROW, 1964. The number of alleles that can be maintained in a finite population, Genetics 49, 725-738.

M. KIMURA AND T. OHTA, 1973. The age of a neutral mutant persisting in a finite population, Genetics 75, 199-212.

J. F. C. KIngman, 1961a. A matrix inequality, Quart. J. Math. Oxford Ser. 12, 78-80.

, 1961b. A mathematical problem in population genetics, Proc. Cambridge Philos. Soc. 57, 574-582.

1976. Coherent random walks arising in some genetical models, Proc. Roy. Soc. London Ser. A 351, 19-31. , 1980. The mathematics of genetic diversity, CBMS-NSF Regional Conf. Ser. in Appl. Math., vol. 34, SIAM, Philadelphia, Pa. ,1982a. On the genealogy of large populations, J. Appl. Probab. 19A, 27-43. , 1982b. The coalescent, Stochastic Process. Appl. 13, 235-248.

V. A. Kostitzin, 1937. Biologie mathématique, A. Colin, Paris.

R. LANDE, 1957. The maintenance of genetic variability by mutation in a polygenic character with linked loci, Genet. Res. 26, 221-235.

S. LESSARD, 1984. Evolutionary dynamics in frequency dependent two-phenotype models, Theoret. Population Biol. (in press). 
R. C. Lewontin, L. R. Ginzburg AND S. D. Tuluapurkar, 1978. Heterosis as an explanation for large amounts of genic polymorphism, Genetics 88, 149-169.

A. J. LOTKA, 1925. Elements of physical biology, Williams and Wilkins, Baltimore, Md.

YU. I. LyUBich, G. D. MAISTROVSKII AND YU. G. OL'KHOVSKII, 1980. Selection-induced convergence to equilibrium in a single-locus autosomal population, Problems Inform. Transmission 16, 66-75.

R. MACARTHUR, 1970. Species packing and competitive equilibrium for many species, Theoret. Population Biol. 1, 1-11.

G. MalÉcor, 1948. Les mathématiques de l'hérédité, Masson, Paris. 1966. Probabilités et hérédité, Presses Univ. de France.

C. MATESSI AND S. D. JAYAKAR, 1976a. Models of density-frequency dependent selection for the exploitation of resources. I: Intraspecific competition, Population Genetics and Ecology (S. Karlin and E. Nevo, eds.), Academic Press, New York, pp. 707-721.

, 1976b. Conditions for the evolution of altruism under Darwinian selection, Theoret. Population Biol. 9, 360-387.

J. MAYNARD SMITH, 1974. The theory of games and the evolution of animal conflicts, J. Theoret. Biol. 74, 209-221.

1978. The evolution of sex, Cambridge Univ. Press.

1981. Will sexual population evolve to an ESS?, Amer. Natur. 117, 1015-1018. 1982. Evolution and the theory of games, Cambridge Univ. Press, Cambridge.

J. Maynard Smith and G. R. Price, 1973. The logic of animal conflicts, Nature (London) 246, 15-18.

E. MAYR, 1963. Animal species and evolution, Harvard Univ. Press, Cambridge, Mass.

R. E. MichOD, 1982. The concept of fitness in population genetics and sociobiology, King's College Sociobiology Group: Current Problems in Sociobiology, Cambridge Univ. Press.

P. A. P. MORAN, 1975. Wondering distributions and the electrophoretic profile, Theoret. Population Biol. 8, 318-330.

1976. Wondering distributions and the electrophoretic profile. II, Theoret. Population Biol. 10, 145-149.

U. Motro, 1982. Optimal rates of dispersal. I, II, Theoret. Population Biol. 21, 394-429.

H. J. MulleR, 1929. The gene as the basis of life, Proc. Internat. Congr. Plant. Sci. 1, 897-921.

T. NAGYLAKI, 1978. The relations between distant individuals in geographically structured populations, Studies in Mathematical Biology, Vol. 15 (S. A. Levin, ed.), Math. Assoc. Amer., Wash., D. C., pp. 588-624.

M. NeI AND K. ROYCHOUdBURY, 1982. Genetic relationships and evolution of races, Evolution Biology, Vol. 14, Plenum Press, New York, pp. 1-56.

U. NUR, 1974. The expected changes in the frequency of alleles affecting the sex ratio, Theoret. Population Biol. 5, 143-147.

T. OHTA AND M. KIMURA, 1973. A model of mutation appropriate to estimate electrophoretically detectable alleles in a finite population, Gen. Res. Camb. 22, 201-204.

A. G. PAKes AND S. TAVARÉ, 1981. Comments on the age distribution of Markov processes, Adv. in Appl. Probab. 13, 681-703.

K. PEARSON, 1904. On a generalized theory of alternative inheritance, with special references to Mendel's laws, Philos. Trans. Roy. Soc. Ser. A 203, 53-86.

F. Proschan, 1965. Peakedness of distributions of convex combinations, Ann. of Math. Statist. 36, 1703-1706.

J. Roughgarden, 1979. Theory of population genetics and evolutionary ecology. An introduction, Macmillan, New York.

P. A. SAmuelson, 1976. Time symmetry and asymmetry in population and deterministic dynamic systems, Theoret. Population Biol. 9, 82-122.

R. F. SHAW, 1958. The theoretical genetics of the sex ratio, Genetics 93, 149-163.

R. F. Shaw AND J. D. MohleR, 1953. The selective advantage of the sex ratio, Amer. Natur. 87, 337-342.

G. G. Simpson, 1953. The major features of evolution, Columbia Univ. Press, New York.

G. L. StebBins, 1950. Variation and evolution in plants, Columbia Univ. Press, New York.

S. TAVARÉ, 1984. Line of descent and genealogical processes, and their applications in population genetics models, Theoret. Population Biol. 25 (to appear). 
M. TURELl, 1984. Heritable genetic variation via mutation-selection balance. Lerch's zeta meets the abdominal bristle, Theoret. Population Biol. 25 (to appear).

M. K. UYenoyAmA AND B. O. BENGTSSON, 1979. Towards a genetic theory for the evolution of the sex ratio, Genetics $93,721-736$.

V. VolterRa, 1931. Lecons sur la théorie mathématique de la lutte pour la vie, Gauthier-Villars, Paris.

B. Wallace, 1981. Basic population genetics, Columbia Univ. Press, New York.

G. A. WATTERSON, 1975. On the number of segregating sites in genetic models without recombination, Theoret. Population Biol. 7, 256-276. , 1984. Lines of descent and the coalescent, Theoret. Population Biol. 25 (to appear).

G. C. Williams, 1975. Sex and evolution, Princeton Univ. Press, Princeton, N. J.

1979. The question of adaptive sex ratio in outcrossed vertebrates, Proc. Roy. Soc. London Ser. B 205, 567-580.

E. O. Wilson, 1975. Sociobiology, the new synthesis, Belknap Press of Harvard Univ. Press, Cambridge, Mass.

S. WRIGHT, 1921. Systems of mating. I: The biometric relations between parent and offspring, Genetics 6, 111-123.

1923a. Mendelian analysis of pure breeds of livestock. II: The Duchess family of shorthorns as bred by Thomas Bates, J. Hered. 14, 405-422.

1923b. The theory of path coefficients, Genetics 8, 239-255.

, 1931. Evolution in Mendelian populations, Genetics 16, 97-159.

1955. Classification of the factors of evolution, Cold Spring Harbor Sympos. Quantitative Biology 20, 16-24.

1968. Evolution and the genetics of populations, Vol. I: Genetic and biometric foundations, Univ. of Chicago Press, Chicago, Ill.

V. C. WYNNE-EDWARDS, 1962. Animal dispersion in relation to social behavior, Hofner, New York.

E. C. Zeeman, 1980. Population dynamics from game theory, Global Theory of Dynamical Systems, Lecture Notes in Math., Vol. 819, Springer-Verlag, Berlin, pp. 471-497. 1981. Dynamics of the evolution of animal conflicts, J. Theoret. Biol. 89, 249-270R.

DePartment of Mathematics, Stanford UNIVERSity, StaNFord, California 94305 
